\title{
Taxonomic review of Gasterophilus (Oestridae, Gasterophilinae) of the world, with updated nomenclature, keys, biological notes, and distributions
}

\author{
Xin-Yu Li ${ }^{1,2}$, Thomas Pape ${ }^{2}$, Dong Zhang'
}

I School of Ecology and Nature Conservation, Beijing Forestry University, Qinghua east road 35, Beijing 10083, China 2 Natural History Museum of Denmark, University of Copenhagen, Universitetsparken 15, Copenhagen, Denmark

Corresponding author: Dong Zhang (ernest8445@163.com)

Academic editor: R. Meier | Received 6 August 2019 | Accepted 22 October 2019 | Published 21 November 2019

http://zoobank.org/84BE68FC-AA9D-4357-9DA0-C81EEBA95E13

Citation: Li X-Y, Pape T, Zhang D (2019) Taxonomic review of Gasterophilus (Oestridae, Gasterophilinae) of the world, with updated nomenclature, keys, biological notes, and distributions. ZooKeys 891: 119-156. https://doi. org/10.3897/zookeys.891.38560

\begin{abstract}
A taxonomic review of Gasterophilus is presented, with nine valid species, 51 synonyms and misspellings for the genus and the species, updated diagnoses, worldwide distributions, and a summary of biological information for all species. Identification keys for adults and eggs are elaborated, based on a series of new diagnostic features and supported by high resolution photographs for adults. The genus is shown to have its highest species richness in China and South Africa, with seven species recorded, followed by Mongolia, Senegal, and Ukraine, with six species recorded.
\end{abstract}

\section{Keywords}

biology, distribution, horse stomach bot fly, identification, nomenclature, taxonomy

\section{Introduction}

The oestrids or bot flies (Oestridae) are known as obligate parasites of mammals in their larval stage. They are often highly host specific, and the short-lived, non-feeding adult flies may show remarkable patterns of camouflage or mimicry (Zumpt 1965; Grunin

Copyright Xin-Yu Li et al. This is an open access article distributed under the terms of the Creative Commons Attribution License (CC BY 4.0), which permits unrestricted use, distribution, and reproduction in any medium, provided the original author and source are credited. 
1965, 1966, 1969; Guimarães and Papavero 1999; Colwell et al. 2006). Species of Gasterophilus Leach (Diptera: Oestridae, Gasterophilinae) are commonly known as horse stomach bot flies (from Greek: gaster for stomach, -philus indicating love or fondness). They have adapted to a larval life in the alimentary tract of Equidae (Zumpt 1965; Grunin 1969; Colwell et al. 2006), and their presence can lead to serious injuries or even death of the host (Hall and Wall 1995; Sequeira et al. 2001; Colwell et al. 2006; Bezdekova et al. 2007; Getachew et al. 2012). Because of their great veterinary importance, Gasterophilus species have received considerable attention since the early 1800s (Clark 1815; Dove 1918; Patton 1937; Zumpt and Paterson 1953; James 1974; Otranto et al. 2005a, b; Colwell et al. 2006, 2007; Zhang et al. 2016; Liu et al. 2016; Huang et al. 2017; Li et al. 2018). A total of more than 40 species-group names have been proposed for what is here recognized as nine valid species because of extensive intraspecific variation (Zumpt 1965; Grunin 1969; Pont 1980; Soós and Minár 1986; Cogley 1991a), and a series of misidentifications can be ascribed to their similar larval morphology (Colwell et al. 2007; $\mathrm{Li}$ et al. 2018). Zumpt (1965) and Grunin (1969) provided the basis of Gasterophilus taxonomy, and further taxonomic studies have been successively published, such as the recognition of $G$. lativentris (Brauer) as a synonym of $G$. pecorum (Fabricius) (Cogley $1991 \mathrm{~b}$ ) and the resurrection of G. flavipes (Olivier) [from synonymy with G. haemorrhoidalis (Linnaeus)] as a valid species (Li et al. 2019). Consequently, an update of the taxonomy, biology and distribution of Gasterophilus species was in demand.

Gasterophilus species were restricted to the Palaearctic and Afrotropical Regions, along with their equid hosts (Zumpt 1965; Leite et al. 1999), before becoming near cosmopolitan due to the association of several species with domestic hosts (Brauer 1863; Dove 1918; Zumpt 1965; Grunin 1969; Pont 1973; James 1974; Soós and Minár 1986; Wood 1987; Xue and Wang 1996; Colwell et al. 2006). Nonetheless, G. meridionalis (Pillers \& Evans) and G. ternicinctus Gedoelst appear to be endemic to the Afrotropics, apparently exclusively associated with Burchell's zebra (Equus quagga burchellii Gray) (Zumpt 1965); and G. nigricornis (Loew) is only recorded from eastern Europe and Central Asia in the Palaearctic Region (Zumpt 1965; Grunin 1969; Soós and Minár 1986; Xue and Wang 1996; Huang et al. 2017; Li et al. 2018). Records of G. meridionalis larvae in domestic horses from the Palaearctic Region (i.e., Iran, Italy, and Turkey) (Özdal et al. 2010; Mashayekhi and Ashtari 2013; Pilo et al. 2015) are suspected to be misidentifications ( $\mathrm{Li}$ et al. 2018).

The life history of Gasterophilus species has been extensively investigated (Clark 1815; Dove 1918; Hadwen and Cameron 1918; Zumpt 1965; Grunin 1969; Catts 1979; Cogley and Cogley 2000; Anderson 2006; Colwell et al. 2006). The adults are known to live only 3-5 days, hovering around the host for ovipositing or gathering at hilltop aggregation sites for mating (Catts 1979). Females lay eggs directly on the host, attaching their eggs to the hairs of the lips, chin, cheeks, or forelegs, depending on the species (Dove 1918; Hadwen and Cameron 1918; Anderson 2006; Colwell et al. 2006; Wood 2006). One exception is $G$. pecorum, which attaches eggs to the tips of grass blades (Zumpt 1965; Grunin 1969). Larvae hatch spontaneously within 5-8 days, or when they are stimulated by moisture and friction associated with host lick- 
ing, feeding or grooming. First instar larvae quickly penetrate into the host around the hatching site and migrate subcutaneously to the hosts' mouth except for G. nasalis, which migrates on the mucosal surface to reach the inter-dental spaces (Zumpt 1965; Anderson 2006; Colwell et al. 2006). Each species of Gasterophilus has a specific site of penetration of the skin and route of migration to the stomach or intestine, where the second and third instar larval development is completed (Cogley et al. 1982; Colwell et al. 2006). It takes about 11 months for the larva to develop, with the third instar taking around 9-10 months in temperate climates. Mature larvae will be excreted with the feces and pupate in the soil (Zumpt 1965; Grunin 1969). The adults eclose after about 2-5 weeks and mate very soon after (Zumpt 1965; Anderson 2006).

Here, we take the opportunity to present an updated catalogue of all nine Gasterophilus species, including revised keys for eggs and adults, and updated diagnoses, host data, distributions, and original as well as major secondary literature for each species. This will be a help for entomologists, veterinarians, and other researchers with an interest in Gasterophilus to familiarize themselves more rapidly and more confidently in the taxonomy, biology, distribution, and literature on this group.

\section{Materials and methods}

\section{Specimens}

Label data provided under 'Material examined' are given in a standardized notation, with country names in capital letters and Chinese provinces in bold. Specimens studied or otherwise referred to are deposited in the following institutions:

IOZ Institute of Zoology, Chinese Academy of Sciences, Beijing, China

KZNM KwaZulu-Natal Museum, Pietermaritzburg, South Africa

MBFU Beijing Forestry University, Beijing, China

MNHN Museum national d'Histoire naturelle, Paris, France

NHMUK Natural History Museum, London, United Kingdom

NHMD Natural History Museum of Denmark, University of Copenhagen, Denmark

NHMW Naturhistorisches Museum Wien, Austria

ZIN Zoological Institute, Russian Academy of Sciences, St. Petersburg, Russia

\section{Imaging and terminology}

A Visionary Digital Imaging System, with a Canon EOS 7D camera (Canon, Inc., Tokyo, Japan) was used to take series of photographs at the Natural History Museum of Denmark. Superimposed photographs were stacked using the Zerene Stacker software and composed using Adobe Photoshop CS6 (Adobe Systems, Inc., San Jose, CA, U.S.A.) on a Windows 10 platform. 
Photographs are provided for $G$. intestinalis (De Geer), G. meridionalis, G. nasalis (Linnaeus), G. nigricornis, G. ternicinctus, and G. pecorum. High resolution photographs of $G$. flavipes, $G$. haemorrhoidalis and $G$. inermis (Brauer) were recently provided by Li et al. (2019).

Morphological terminology follows Cumming and Wood (2009) for adults and Ferrar (1987) for eggs.

\section{Distribution}

A worldwide species diversity map was produced using the non-commercial version of StatPlanet (StatSilk 2018).

\section{Format of catalog}

Regional catalogues (Pont 1973, 1980; Soós and Minár 1986) are followed with regard to synonyms as the valid names for species of Gasterophilus are accepted throughout current literature and the synonymies appear stable. All original proposals of available and unavailable names and first occurrences of misspellings were checked and updated for information on type locality. Generic synonyms are given with author, year: page, type species and mode of designation. The most important taxonomic, morphological, biological, distributional and evolutionary studies of Gasterophilus are selected and listed chronologically.

Valid species are treated in alphabetic order, with the valid name given in bold followed by a list of all synonyms in their original generic combination with author, year and page plus type locality given in modern English (with an original quotation where considered relevant, e.g., France, Pyrenees, "Dans les Pyrénées"). Precise localities provided by early authors are cited as well [e.g. Democratic Republic of the Congo (as "Zaire"), $11.5 \mathrm{~km} \mathrm{~W}$ of Luapula river (as "6 milles W. du Luapula")]. Synonyms are listed chronologically for each species, followed by all published misspellings known to us. Most important references about taxonomic, morphological, biological, distributional and evolutionary studies of species in Gasterophilus are selected and listed chronologically.

Host records and distribution are given based on information from specimens examined for the present study (directly or from photos) and data from Brauer (1863), Zumpt (1965), Guimarães (1967), Grunin (1969), Pont (1973), James (1974), Kaboret et al. (1986), Soós and Minár (1986), Pearse et al. (1989), Pandey et al. (1992), Escartin and Bautista (1993), Xue and Wang (1996), Güiris et al. (2010), Özdal et al. (2010), Tavassoli and Bakht (2012), Mashayekhi and Ashtari (2013), Pape (2013), Ganjali and Keighobadi (2016), Huang et al. (2016), Hoseini et al. (2017), Muller and Ranwashe (2017), Tähtinen and Lahti (2017), van Noort and Ranwashe (2017). Host data is listed alphabetically, with both common name and scientific name. Distribution is given with countries listed alphabetically in their respective biogeographical regions, i.e., Afrotropi- 
cal, Australasian, Nearctic, Neotropical, Palaearctic and Oriental Regions with boundaries as applied in Pape (1996). Further information, like Provinces or States, were given for countries with large continental area (i.e. Argentina, Australia, Brazil, Canada, Chile, China, United States of America), if applicable. Large islands (i.e., Corsica, Sardinia and Sicily) are listed together with their mainland countries. Non-vouchered literature records of G. flavipes obtained from Li et al. (2019) were retained with a question mark.

Biological information provided for eggs, larvae and adults is summarized and presented in Table 1.

The generic diagnosis is provided for adults, eggs and larvae, while species diagnoses are provided only for adults. Keys are modified from already existing keys and updated with more diagnostic characters for both adults and eggs. Comprehensive identification keys to first instar larvae were published by Grunin (1969) and Zumpt (1965), and to third instar larvae by Li et al. (2018).

\section{Catalogue}

\section{Genus Gasterophilus}

Figs 1-19; Table 1

Gasterophilus Leach, 1817: 2. Type species: Oestrus equi Clark, 1797 [= Oestrus intestinalis De Geer, 1776], by subsequent designation of Curtis (1826: 146).

Gastrus Meigen, 1824: 174. Type species: Oestrus intestinalis De Geer, 1776, by subsequent designation of Coquillett (1910: 546).

Gastrophilus Agassiz, 1846: 160. Unjustified emendation of Gasterophilus Leach, 1817. Type species: Oestrus equi Clark, 1797 [= Oestrus intestinalis De Geer, 1776] (automatic).

Enteromyza Rondani, 1857: 20. Unnecessary new replacement name for Gastrus Meigen, 1824 and Gasterophilus Leach, 1817. Type species: Oestrus equi Clark, 1797 [= Oestrus intestinalis De Geer, 1776] (automatic).

Rhinogastrophilus Townsend, 1918: 152. Type species: Oestrus nasalis Linnaeus, 1758, by original designation.

Enteromyia Enderlein, 1934: 425. Type species: Oestrus haemorrhoidalis Linnaeus, 1758, by original designation.

Stomachobia Enderlein, 1934: 425. Type species: Oestrus pecorum Fabricius, 1794, by original designation.

Haemorrhoestrus Townsend, 1934: 406. Type species: Oestrus haemorrhoidalis Linnaeus, 1758 , by original designation.

Progastrophilus Townsend, 1934: 406. Type species: Oestrus pecorum Fabricius, 1794, by original designation.

Selected references. Brauer (1863: 53); Zumpt (1965: 111); Grunin (1969: 21); Pont (1973: 698); James (1974: 92); Kettle (1974); Papavero (1977: 19); Wood (1987: 


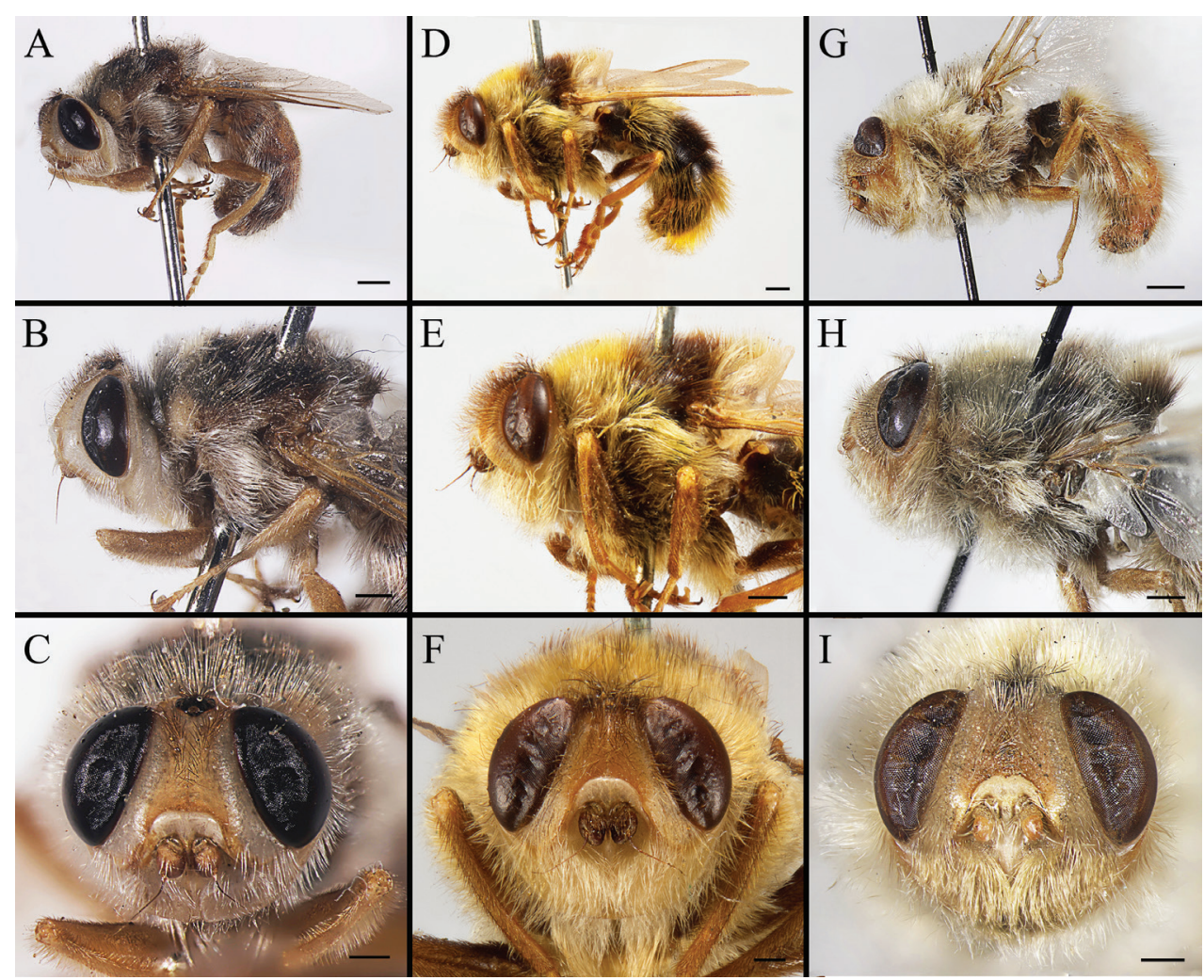

Figure I. Left lateral view of habitus $(\mathbf{A}, \mathbf{D}, \mathbf{G})$, head and thorax $(\mathbf{B}, \mathbf{E}, \mathbf{H})$, and head in frontal view (C, F, I) of male Gasterophilus species, modified from Li et al. (2019) A-C G. flavipes (Olivier); Morocco (in IOZ) D-F G. haemorrhoidalis (Linnaeus); China (in MBFU) G-I G. inermis (Brauer); Germany (in NHMD). Scale bars: $1 \mathrm{~mm}(\mathbf{A}, \mathbf{D}, \mathbf{G}) ; 0.5 \mathrm{~mm}(\mathbf{B}, \mathbf{C}, \mathbf{E}, \mathbf{F}, \mathbf{H}, \mathbf{I})$.

1148); Soós and Minár (1986: 238); Xue and Wang (1996: 2209); Pape (2001); Pape et al. (2017); Otranto et al. (2005); Colwell et al. (2006: 5); Colwell et al. (2007); Felix et al. (2007); Zhang et al. (2012); Huang et al. (2016); Zhang et al. (2016); Li et al. (2018, 2019); Yan et al. (2019).

Diagnosis. Body covered with dense, yellowish hair-like setae, variously interrupted by reddish-yellow or dark brown (or black) bands (Figs 1-10). Facial plate with a narrow median keel. Antennal arista long, slender, gradually tapered and slightly flattened, with short, sparse microtrichia (Figs 1C, F, I, 2C, F, I, 3C, F, I, 7C, F, I, 8C, F, I, 9C, F, I). Proboscis and palpus vestigial, visible as small, yellow or brown knobs. Thorax ground color mainly dark brown or black (Figs 4-6, 7A, D, G, 8A, D, G, 9A, D, G). Notopleuron weakly defined. Posterior spiracle open, with short, hair-like fringes, lappets oriented obliquely at an angle of about 45 degrees. Wing vein $\mathrm{M}$ almost straight, very slightly curved posteriorly; vein $\mathrm{A}_{1}+\mathrm{Cu} \mathrm{A}_{2}$ extending to wing margin (Fig. 10). Upper and lower calypters yellowish, fringed with long, whitish, hair-like setae along the external margin. Abdomen ground color yellow, dark brown 


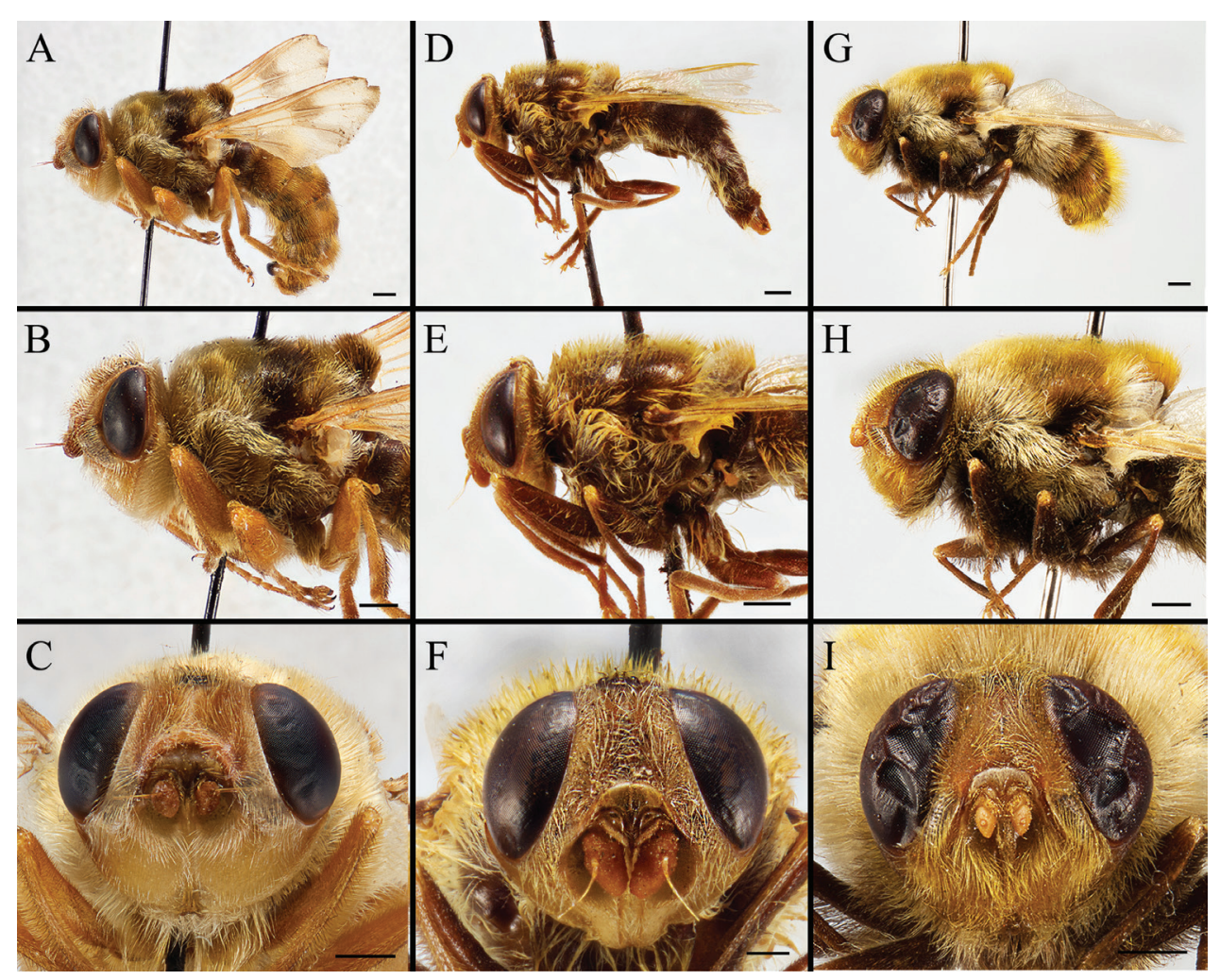

Figure 2. Left lateral view of habitus $(\mathbf{A}, \mathbf{D}, \mathbf{G})$, head and thorax $(\mathbf{B}, \mathbf{E}, \mathbf{H})$, and head in frontal view $(\mathbf{C}, \mathbf{F}, \mathbf{I})$ of Gasterophilus species A-C Male G. intestinalis (De Geer) China (in MBFU) D-F Female G. meridionalis (Pillers \& Evans); South Africa (in KZNM) G-I Male G. nasalis (Linnaeus) China (in MBFU). Scale bars: $1 \mathrm{~mm}(\mathbf{A}-\mathbf{E}, \mathbf{I}) ; 0.5 \mathrm{~mm}(\mathbf{F})$.

or black, sometimes with several irregular dark spots (Figs 1A, D, G, 2A, D, G, 3A, D, G, 4A, C, E, 5A, C, E, 6A, C, E, 7A, B, D, E, 8A, B, D, E, 9A, B, D, E, G, H). Male cercus (Figs 11-13) broadly connected to its counterpart by a membrane at the base, with a long or short free apex (Figs 11C, F, I, 12C, F, 13C, F, I); surstylus with a rounded or gradually tapered apex (Figs 11B, E, H; 12B, E; 13B, E, H); phallus short, dorsolateral processes of distiphallus reduced, epiphallus absent; pregonite tuberculous; postgonite falcate (Figs 11A, D, G, 12A, D, 13A, D, G); processi longi (remnants of sternite 10) setose, tubercular or elongated (Patton 1937, Grunin 1969). Female terminalia (Figs 14-16) gradually tapered, either short and straight (Fig. 9E) or long and curved forward (Figs 7B, E, H, 8B, E, 9B, H); segment 7 modified, fully sclerotized, tube-shaped, dorsally with a longitudinal suture, without separation of tergite and sternite 7; tergite 8 laterally expanded downwards; sternite 8 either with a longitudinal concavity in the middle and with a keel-shaped apex (Fig. 16F), or longitudinally ridged in the middle and with a scallop-shaped apex (Figs 14C, F, L, 15C, F, L, 16C); tergite 10 (epiproct) composed of two approximately triangular sclerites 


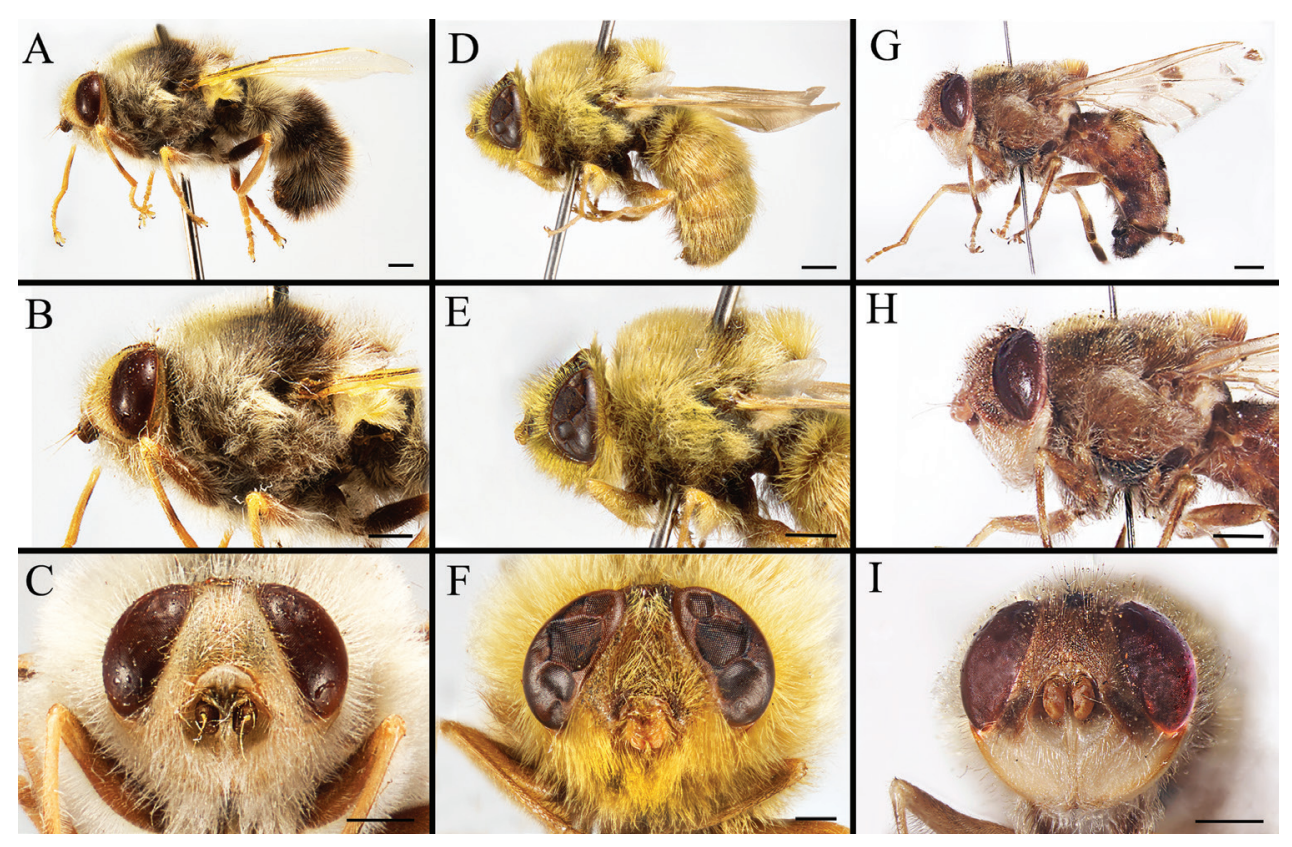

Figure 3. Left lateral view of habitus $(\mathbf{A}, \mathbf{D}, \mathbf{G})$, head and thorax $(\mathbf{B}, \mathbf{E}, \mathbf{H})$, and head in frontal view (C, F, I) of male Gasterophilus species A-C G. nigricornis (Loew); China (in MBFU) D-F G. pecorum (Fabricius); China (in MBFU) G-I G. ternicinctus Gedoelst; South Africa (in MBFU). Scale bars: $1 \mathrm{~mm}(\mathbf{A}-\mathbf{I})$.

(Figs 14B, E, H, 15B, E, H, 16B, E); cercus long and narrow, narrowly connected to its counterpart by membrane and with a very short prolongation (Figs 14A, D, G, $15 \mathrm{~A}, \mathrm{D}, \mathrm{G}, 16 \mathrm{~A}, \mathrm{D})$. Eggs with an attachment organ, short and posteriorly located or elongated and situated ventrally (Figs 17-18). The larva with a bilobed, highly constricted pseudocephalon, three thoracic segments, seven abdominal segments, and the anal division divided into three subdivisions (Zumpt 1965; Grunin 1969; Li et al. 2018). The freshly hatched larva fusiform, anteriorly encircled with strong body spinose; posterior spiracles slightly or distinctly elongated, fully exposed, with two serrated margined slits (Zumpt 1965; Grunin 1969). The second and third instar larva sub-cylindrical, with mouth hooks posterolaterally curved and sharply pointed, and a pair of oral plates between mouth hooks; most of the body segments circled anteriorly by strong, posteriorly directed spines arranged in one, two or three rows (Zumpt 1965; Grunin 1969; Li et al. 2018). The third instar larva distinctively colored in red, yellow or green (Li et al. 2018).

Hosts. Known exclusively from the genus Equus Linnaeus (Perissodactyla: Equidae). So far, no records have been made from the species E. grevyi Oustalet (Grévy's zebra) and E. kiang Moorcroft (kiang or Tibetan wild ass).

Distribution and diversity. Native distribution matches that of the horse family, currently with highest diversity in China and South Africa, with 7 species recorded, followed by Mongolia, Senegal and Ukraine, with 6 species recorded (Fig. 19). Introduced with domestic hosts to most parts of the world. 


\section{Key to adults of Gasterophilus spp.}

1 Wing with darkened patches (Fig. 10C, D, H, I) ………………………......... 2

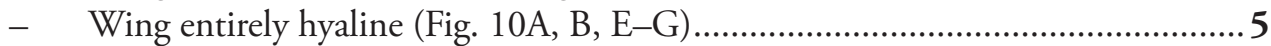

2 Wing patches sharply demarcated (Fig. 10I); hind tibia and tarsus distinctly flattened (to a lesser degree in female), tarsomeres 2-4 shortened, as broad as long or broader than long (Figs 3D, 9G, H) Gasterophilus ternicinctus

- Wing patches with ill-defined edges (Figs 10C, D, H); hind tibia and tarsus unmodified, tarsomeres 2-4 long and narrow, distinctly longer than broad ............. 3

3 Antennal pedicel elongated, length/width ratio more than 0.8 (Figs 3F, 9F); facial plate setose; abdomen ground color yellow in male (Fig. 3D), mainly black in female (Fig. 9E); female terminalia short (Fig. 9E), abdominal sternite 8 with a keel-shaped apex (Fig. 16F)

Gasterophilus pecorum

- Antennal pedicel short, length/width ratio less than 0.5; facial plate bare; abdomen ground color mainly yellow; female terminalia elongated, abdominal sternite 8 with a scallop-shaped apex (Figs 15C, F, I, 16C) ........................................... 4

4 Hind trochanter ventrally with a spatulate process in male or a tubercle in female (Grunin 1969: fig. 95); male surstylus yellow, with a black apex (Fig. 12A-C); female abdominal segment 7 longer than broad (Fig. 8B)

Gasterophilus intestinalis

- Hind trochanter without a process or tubercle; male surstylus entirely yellow (Grunin 1969: fig. 86); female abdomen abdominal segment 7 broader than long (Fig. $7 \mathrm{H}$ )

Gasterophilus inermis

5 Crossvein dm-cu present; antennal postpedicel yellow or brownish; meral setae

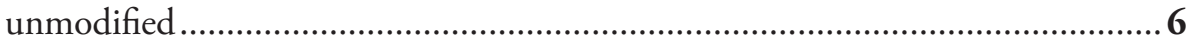

- Crossvein dm-cu absent (Fig. 10G); antennal postpedicel red-brown to blackish (Figs 3C, 9C); meral setae with tip swollen.................. Gasterophilus nigricornis

6 Crossvein dm-cu distinct; antennal postpedicel globular .....................................

- Crossvein dm-cu extremely faint (Fig. 10E); antennal postpedicel long-oval (Fig. 2F)

Gasterophilus meridionalis

7 Distance between crossveins $\mathrm{r}-\mathrm{m}$ and $\mathrm{dm}-\mathrm{cu}$ at least twice as long as $\mathrm{r}-\mathrm{m}$; male cercus short and broad, length/width ratio equal to or less than 1.0, surstylus much

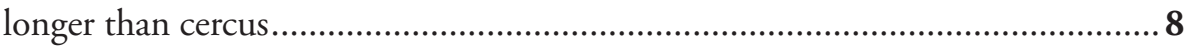

- Distance between crossveins $\mathrm{r}-\mathrm{m}$ and $\mathrm{dm}$-cu less than length of $\mathrm{r}-\mathrm{m}$ (Fig. 10F); male cercus long and narrow, length/width ratio more than 3.0, surstylus and cercus of similar length (Fig. 12D-F)

Gasterophilus nasalis

8 Postsutural scutum with a light (yellowish), rectangular area near scutoscutellar suture (Fig. 4A); legs yellow; abdomen ground color yellow, covered with yellow setae (Figs 1A, 4A, 7A-B); male with surstylus gradually tapered proximally and distally, surstylar setae long, reaching the sagittal plane (Fig. 11A-C) Gasterophilus flavipes

- Postsutural scutum with ground color uniformly brown or black (Figs 4C, D, 7D); legs yellowish brown, with femora distinctly darkened; abdomen ground color dark brown or black, with reddish-yellow or orangish setae posteriorly (Figs 1D, 7E); male with surstylus abruptly tapered distally, surstylar setae short, reaching at most halfway to the sagittal plane (Fig. 11D-F) 


\section{Key to eggs of Gasterophilus spp.}

1 Posteriorly with an elongated pedicel (a continuation of the broad chorionic flanges) (Fig.17A, B, E, F) ........................................................................ 2

- Posteriorly with a very short pedicel or without a pedicel (Figs 17I, J, M, N, 18A,

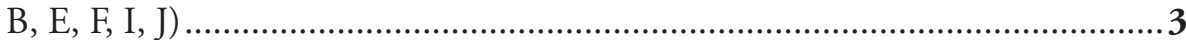

2 Pedicel short and thick, with width/length ratio around 1/4 in lateral view, accounting for $1 / 3$ of the total egg length (Fig. 16A-D) .................. Gasterophilus flavipes

- Pedicel long and slender, with width/length ratio around 1/6 in lateral view, accounting for $2 / 5$ of the total egg length (Fig. 17E-H)

Gasterophilus haemorrhoidalis

3 Chorion brownish black, posteriorly with a short attachment organ, accounting for $1 / 6$ of egg length (Fig. 18I-L) Gasterophilus pecorum

- Chorion yellowish, ventrally with a long attachment organ, accounting for at least $1 / 2$ of egg length ................................................................................. 4

4 Egg gradually tapered, anterior half distinctly broader than posterior half (Fig. 17M-P) Gasterophilus intestinalis

- Egg fusiform, swollen in the middle, anteriorly and posteriorly tapered ............5

5 Attachment organ around half the length of the egg (Cogley 1991b: fig. 8) Gasterophilus ternicinctus

- Attachment organ almost the same length as the egg. .6

6 Operculum placed apically (parallel to the egg's cross section) (Fig. 18A)

Gasterophilus nasalis

- Operculum placed sub-apically (distinctly angled relative to the egg's cross section) (Figs 17I, 18E)

7 Micropylar position apical (on top surface) (Cogley 1991b: fig. 9)

Gasterophilus meridionalis

- Micropylar position sub-apical (on ventral surface) 8

8 Operculum length/width ratio about 2.0 (Fig. 18E) Gasterophilus nigricornis

- Operculum length/width ratio about 4.0 (Fig. 17I) Gasterophilus inermis

\section{Gasterophilus flavipes (Oliver, 1811)}

Figs 1A-C, 4A, B, 7A-C, 10A, 11A-C, 14A-C, 17A-D; Table 1

Oestrus flavipes Olivier, 1811: 467. Type locality: France, Pyrenees (“Dans les Pyrénées").

Selected references. Brauer (1863: 80); Patton (1937); Li et al. (2019).

Diagnosis. Facial plate bare. Postsutural scutum of light color (yellowish), with rectangular area near scutoscutellar suture. Wing completely hyaline. Distance between crossveins $\mathrm{r}-\mathrm{m}$ and $\mathrm{dm}-\mathrm{cu}$ at least twice as long as $\mathrm{r}-\mathrm{m}$. Meron with unmodified setae. Legs yellow; hind tarsus with long, strong and dense setae ventrolaterally. Abdomen ground color yellow. Male cercus short and broad, length/width ratio equal or less 


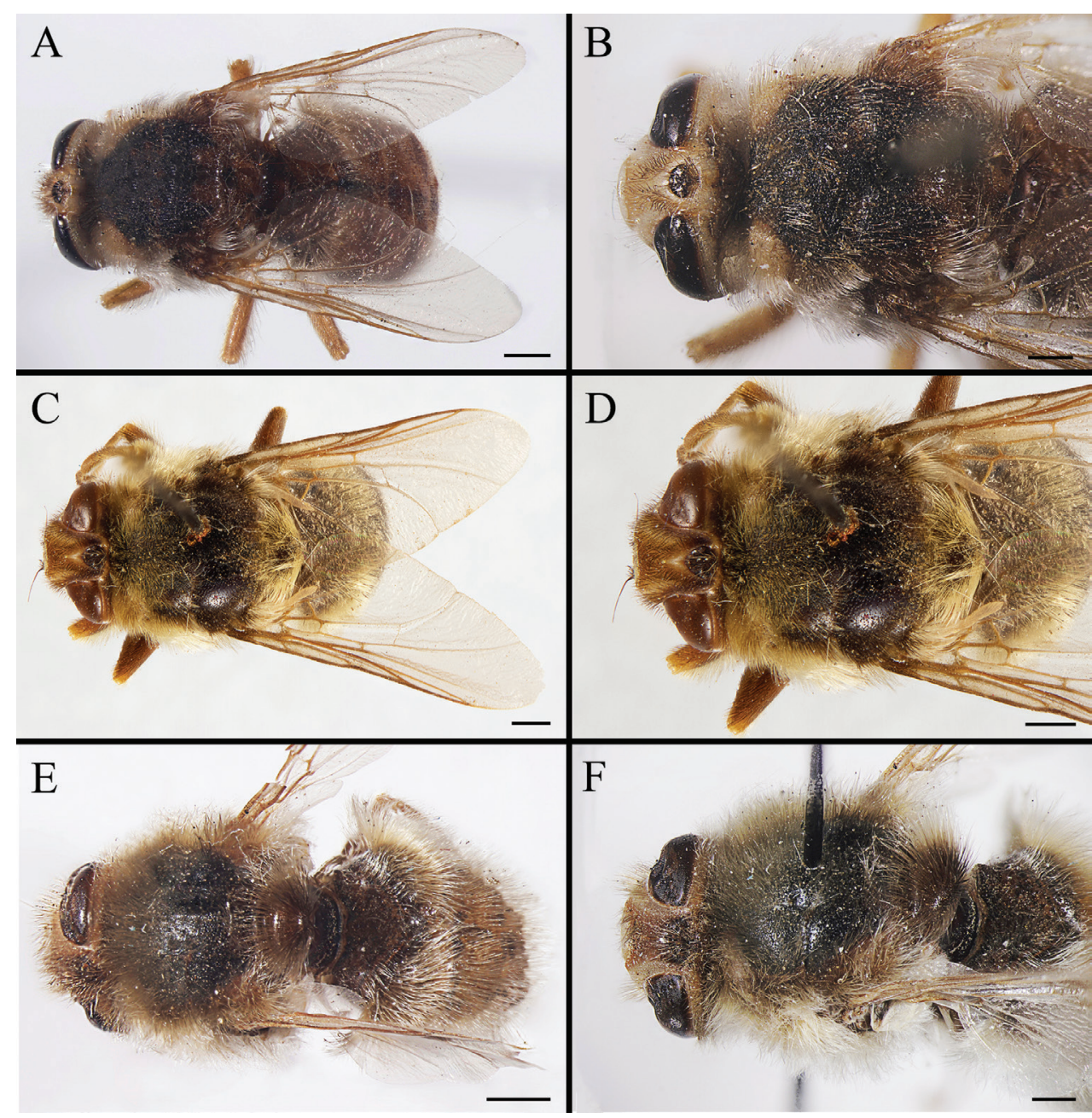

Figure 4. Dorsal view of habitus $(\mathbf{A}, \mathbf{C}, \mathbf{E})$ and head and thorax $(\mathbf{B}, \mathbf{D}, \mathbf{F})$ of male Gasterophilus species, modified from Li et al. (2019) A, B G. flavipes (Olivier) C, D G. haemorrhoidalis (Linnaeus) E, F G. inermis (Brauer). Scale bars: $1 \mathrm{~mm}(\mathbf{A}, \mathbf{C}-\mathbf{D}, \mathbf{E}) ; 0.5 \mathrm{~mm}(\mathbf{B}, \mathbf{F})$.

than 1.0; surstylus yellow, gradually tapered proximally and distally, with a gradually tapered apex; surstylar setae long, reaching the sagittal plane; processi longi tubercular. Female sternite 8 longitudinally ridged in the middle and with a scallop-shaped apex.

Material examined. CHINA - Xinjiang Uyghur Autonomous Region • $10 \overbrace{}^{\lambda}{ }^{\lambda}$, 14우우; Kalamaili, Qiaomuxibai water reservoir; $45^{\circ} 13.8^{\prime} \mathrm{N}, 89^{\circ} 3.0^{\prime} \mathrm{E}$ (DDM);

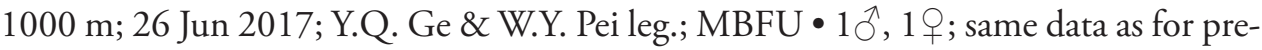
ceding; NHMD. - Inner Mongolia •1 $\delta^{\top}$; Chifeng; 1 Jul. 1960, collector unknown; IOZ $\bullet 1 \delta^{\text {; }}$; same collection locality as for preceding; 3 Jul. 1960; collector unknown; IOZ. CYPRUS • 1 $\hat{\delta}$; no further data; NHMUK. MOROCCO • $1 \hat{\delta}$; no further data; 1897; G. Buchet leg.; MNHN・10̄; Haute Moulouya; 1918; Thullet leg.; MNHN 
Table I. Natural history of Gasterophilus species.

\begin{tabular}{lcccc}
\hline Species & $\begin{array}{c}\text { Embryonic } \\
\text { development } \\
\text { /days }\end{array}$ & $\begin{array}{c}\text { Hatching } \\
\text { strategy }\end{array}$ & $\begin{array}{c}\text { First instar } \\
\text { development }\end{array}$ & $\begin{array}{c}\text { Second and third instar } \\
\text { development }\end{array}$ \\
\hline G. flavipes & NA & NA & NA & NA \\
G. & 2 & $\begin{array}{r}\text { Stimulated } \\
\text { by moisture } \\
\text { from licking } \\
\text { or feeding of } \\
\text { hosts. }\end{array}$ & $\begin{array}{c}\text { - Penetrate epidermis the lips of hosts and } \\
\text { migrate into mouth. }\end{array}$ & $\begin{array}{c}\text { - Second instar move to } \\
\text { stomach and duodenum; } \\
\text { - Third instar larvae become } \\
\text { detached after some time }\end{array}$ \\
& & & $\begin{array}{c}\text { and then pass to the rectum } \\
\text { and re-attach themselves. }\end{array}$
\end{tabular}

G. inermis

G. intestinalis

G. meridionalis

NA

$5-10$

G. nasalis

G. nigricornis

NA
Spontaneous. · Penetrate skin of hosts at hatching site;

- Migrate firstly under epidermis to the corner of mouth and then under the mucous

membrane inside cheek.

5 Stimulated by moisture and friction supplied by rubbing and licking of

hosts. anterior to posterior end; the migration route is almost parallel to the right or left lateral margin of tongue.

$$
\text { NA }
$$$$
\text { NA }
$$

Spontaneous.

$$
\begin{aligned}
& \text { - Migrate on surface to } \\
& \text { inter-dental spaces of }
\end{aligned}
$$
hosts.
- Burrow from the

- Second and third instar larvae found in the rectum.

21-26

- Young second instar larvae attach to the pharynx and the sides of the epiglottis, and then pass to the stomach;

- Third instar larvae are generally found clustered near the boundary of the nonglandular and glandular epithelia.

\footnotetext{
- Attached to stomach mucosa.

- Moult to second instar at inter-dental sites;

- Migrate to duodenum and attach near pylorus.
} migrate firstly under epidermis to the corner of mouth and then under the mucous membrane inside the cheek.

22-28 Domestic donkey;

- Molt to the second stage in 31-34 . Domestic donkey;

the central part of the cheek;

- Migrate to duodenum,

attach to mucosa and

become encysted;

- Third instar larvae leave the · · Mongolian wild ass; cyst and become attached

Pupal Host
period
/days

(Equus africanus asinus

Linnaeus) [speculated

by Brauer (1863)

without evidence].

15-26 . Burchell's zebra $(E$. quagga burchellii Gray);

- Domestic horse (E. ferus caballus Linnaeus)

- Domestic donkey;

- Mongolian wild ass

(E. hemionus hemionus Pallas);

- Mountain zebra $[E$. zebra Linnaeus];

\section{$\times$ Wild horse $(E$}

przewalskii Poliakov)

\section{- Mongolian wild ass; \\ . Wild horse.}

\author{
- Domestic horse; \\ - Mongolian wild ass; \\ . Wild horse.
}
28-31 Burchell's zebra.
16-24 Burchell's zebra;
- Domestic donkey;
- Domestic horse;
- Mongolian wild ass;
. Wild horse. - Wild horse. superficially to the mucous membrane. 


\begin{tabular}{|c|c|c|c|c|c|c|}
\hline Species & $\begin{array}{c}\text { Embryonic } \\
\text { development } \\
\text { /days }\end{array}$ & $\begin{array}{l}\text { Hatching } \\
\text { strategy }\end{array}$ & $\begin{array}{c}\text { First instar } \\
\text { development }\end{array}$ & $\begin{array}{c}\text { Second and third instar } \\
\text { development }\end{array}$ & $\begin{array}{c}\text { Pupal } \\
\text { period } \\
\text { /days }\end{array}$ & Host \\
\hline G. pecorum & $5-8$ & $\begin{array}{c}\text { Stimulated } \\
\text { by moisture } \\
\text { and friction } \\
\text { supplied } \\
\text { by hosts' } \\
\text { ingestion. }\end{array}$ & $\begin{array}{c}\text { - Penetrate mouth } \\
\text { mucosa of hosts; } \\
\text {. Migrate to the soft } \\
\text { palate and at the root of } \\
\text { the tongue, occasionally } \\
\text { the pharynx and } \\
\text { oesophagus. }\end{array}$ & $\begin{array}{l}\text {. Molt to second and third } \\
\text { instar at oral site; } \\
\text {. Third instars migrate to } \\
\text { stomach and attach to } \\
\text { mucosa. }\end{array}$ & $12-21$ & $\begin{array}{c}\text {. Burchell's zebra; } \\
\text { · Domestic donkey; } \\
\text {. Domestic horse; } \\
\text { · Mongolian wild ass; } \\
\text {. Persian onager ( } E \text {. } \\
\text { hemionus onager } \\
\text { Boddaert); } \\
\text {. Wild horse. }\end{array}$ \\
\hline G. ternicinctus & NA & NA & $\cdot \mathrm{NA}$ & $\begin{array}{l}\text { Second and third instar } \\
\text { larvae found in stomach. }\end{array}$ & $20-27$ & × Burchell's zebra. \\
\hline
\end{tabular}

Table I. Continued.

\begin{tabular}{|c|c|c|c|c|c|c|c|}
\hline Species & $\begin{array}{c}\text { Number } \\
\text { of eggs/ } \\
\text { female }\end{array}$ & Mating site & $\begin{array}{c}\text { Oviposition } \\
\text { site }\end{array}$ & $\begin{array}{c}\text { Arrangement } \\
\text { of eggs }\end{array}$ & $\begin{array}{c}\text { Oviposition } \\
\text { behavior }\end{array}$ & $\begin{array}{l}\text { Longevity } \\
\text { /days }\end{array}$ & Major references \\
\hline G. flavipes & NA & $\mathrm{NA}$ & NA & $\mathrm{NA}$ & NA & $\mathrm{NA}$ & $\begin{array}{c}\text { Brauer 1863; Li et } \\
\text { al. } 2019\end{array}$ \\
\hline $\begin{array}{l}\text { G. } \\
\text { haemorrhoidalis }\end{array}$ & $50-200$ & Around host & $\begin{array}{l}\text { Hairs along } \\
\text { the edge of } \\
\text { the lips }\end{array}$ & $\begin{array}{l}\text { One egg per } \\
\text { hair }\end{array}$ & $\begin{array}{l}\text { 'Hit-and-flee': } \\
\text { female in full flight } \\
\text { swiftly collides with } \\
\text { a host and rapidly } \\
\text { deposits an egg } \\
\text { and then flies away } \\
\text { before repeating } \\
\text { the process }\end{array}$ & $1-7$ & $\begin{array}{l}\text { Dove 1918; Zumpt } \\
\text { 1965; Colwell et } \\
\text { al. 2006; Anderson } \\
\text { 2006; Huang et al. } \\
2016\end{array}$ \\
\hline G. inermis & $320-360$ & $\begin{array}{c}\times \text { Topographic } \\
\text { landmark } \\
\text { (Tops of hilltop } \\
\text { shrubs/trees }\end{array}$ & $\begin{array}{l}\text { Base of the } \\
\text { hairs on } \\
\text { cheeks }\end{array}$ & $\begin{array}{l}\text { One egg per } \\
\text { hair }\end{array}$ & Hit-and-flee & $21-26$ & $\begin{array}{c}\text { Zumpt 1965; Colwell } \\
\text { et al. 2006; Anderson } \\
\text { 2006; Huang et al. } \\
2016\end{array}$ \\
\hline G. intestinalis & $400-1000$ & $\begin{array}{l}\times \text { Around host; } \\
\times \text { Topographic } \\
\text { landmark } \\
\text { (hilltop, top of } \\
\text { shrubs/trees }\end{array}$ & $\begin{array}{l}\text { Distal half of } \\
\text { the hairs on } \\
\text { forelegs and } \\
\text { chest }\end{array}$ & $\begin{array}{l}\text { Often several } \\
\text { eggs found on } \\
\text { one hair }\end{array}$ & $\begin{array}{l}\text { Female hovers } \\
\text { slowly in one } \\
\text { spot and quickly } \\
\text { deposits several } \\
\text { eggs before flying } \\
\text { to another position } \\
\text { or to another host }\end{array}$ & $7-21$ & $\begin{array}{c}\text { Dove 1918; Zumpt } \\
\text { 1965; Catts 1979; } \\
\text { Cogley et al. 1982; } \\
\text { Colwell et al. 2006; } \\
\text { Huang et al. } 2016\end{array}$ \\
\hline G. meridionalis & NA & NA & NA & NA & NA & NA & Zumpt 1965 \\
\hline G. nasalis & $300-500$ & Around host & $\begin{array}{l}\text { Hairs under } \\
\text { chin }\end{array}$ & $\begin{array}{l}\text { Usually only } \\
\text { one egg per } \\
\text { hair, but } \\
\text { occasionally } \\
\text { five have been } \\
\text { counted }\end{array}$ & $\begin{array}{l}\text { Hit-and-flee, and } \\
\text { an undisturbed } \\
\text { female may deposit } \\
\text { up to } 20 \text { eggs } \\
\text { without leaving } \\
\text { the host }\end{array}$ & $1-12$ & $\begin{array}{l}\text { Dove 1918; Zumpt } \\
\text { 1965; Colwell et } \\
\text { al. 2006; Anderson } \\
\text { 2006; Huang et al. } \\
2016\end{array}$ \\
\hline G. nigricornis & $350-350$ & NA & $\begin{array}{l}\text { Base of the } \\
\text { hairs on } \\
\text { cheek or } \\
\text { neck }\end{array}$ & $\begin{array}{l}\text { One egg per } \\
\text { hair }\end{array}$ & Hit-and-flee & NA & $\begin{array}{c}\text { Zumpt 1965; Colwell } \\
\text { et al. 2006; Anderson } \\
\text { 2006; Huang et al. } \\
2016\end{array}$ \\
\hline G. pecorum & $1300-2600$ & Around host & $\begin{array}{l}\text { Off host, } \\
\text { mainly on } \\
\text { tip of grass } \\
\text { blades, and } \\
\text { also on plant } \\
\text { stems }\end{array}$ & $\begin{array}{c}\text { In rows } \\
\text { (groups of } \\
10-15 \text { eggs/ } \\
\text { batch }\end{array}$ & $\begin{array}{c}\text { Female } \\
\text { continuously lays } \\
\text { several eggs in one } \\
\text { spot before flying } \\
\text { to another position }\end{array}$ & $1-4$ & $\begin{array}{c}\text { Zumpt 1965; Colwell } \\
\text { et al. 2006; Hoseini } \\
\text { et al. 2017; Huang et } \\
\text { al. } 2016\end{array}$ \\
\hline G. ternicinctus & NA & NA & NA & NA & NA & NA & Zumpt 1965 \\
\hline
\end{tabular}


•1ð̊; Moyen Atlas; Hun. 1949; L. Chopard leg.; MNHN・19; LIBYA • Zuwarah; no further data; NHMUK $\bullet 10$; SUDAN • Ed Dueim; 1937; collector unknown; NHMUK $\bullet 1 \hat{\delta}$; no further data; NHMUK.

Hosts. Donkey (E. africanus asinus Linnaeus) [speculated by Brauer (1863) without evidence].

Distribution. Afrotropical - Sudan. Palaearctic - China (Inner Mongolia, Xinjiang), Croatia?, Cyprus, Egypt?, France, Iran?, Kazakhstan?, Libya, Morocco, Spain?, Turkey?

\section{Gasterophilus haemorrhoidalis (Linnaeus, 1758)}

Figs 1D-F, 4C, D, 7D-F, 10B, 11D-F, 14D-F, 17E-H; Table 1

Oestrus haemorrhoidalis Linnaeus, 1758: 584 (as "hæmorrhoidalis"). Type locality: not given, probably Sweden, Germany, and France (through reference to Fauna Svecica and unspecified works by Johann Leonhard Frisch and Antoine Ferchault de Réaumur).

Oestrus salutiferus Clark, 1816: 3. Type locality: England.

Oestrus duodenalis Schwab, 1840: 35. Type locality: Europe. Proposed in synonymy with Oestrus salutiferus Clark, 1816, made available from subsequent use as a valid name for a taxon by Gistel (1848: 153).

Gastrophilus pallens Bigot, 1884: 4. Type locality: Sudan, Suakin (as "Suakim? Soudan oriental").

Gasterophilus pseudohaemorrhoidalis Gedoelst, 1923: 272 (as "pseudo-haemorrhoïdalis"). Type locality: Eritrea, Asmara (as "Erythree: Asmara"); Republic of the Congo, Katanga Province, Biano (as "Katanga: Biano") and Zambia (as "Zambi").

Oestrus hemorrhoidalis Clark, 1815: 71; incorrect subsequent spelling of haemorrhoidalis Linnaeus, 1758.

Oestrus haemorrhoidales Clark, 1816: [1]; incorrect subsequent spelling of haemorrhoidalis Linnaeus, 1758.

Oestrus hemorroidalis Guérin-Méneville, 1827: 96; incorrect subsequent spelling of haemorrhoidalis Linnaeus, 1758.

Oestrus aemorrhoidalis Rondani, 1857: 21; incorrect subsequent spelling of haemorrhoidalis Linnaeus, 1758.

Selected references. Brauer (1863: 83); Zumpt (1965: 122); Grunin (1969: 40); Pont (1973: 698); James (1974: 97); Soós and Minár (1986: 238); Cogley (1991b); Xue and Wang (1996: 2209); Otranto et al. (2005); Colwell et al. (2006: 9); Colwell et al. (2007); Zhang et al. (2016); Li et al. (2018, 2019); Yan et al. (2019).

Diagnosis. Facial plate bare. Wing completely hyaline. Distance between crossveins $\mathrm{r}-\mathrm{m}$ and $\mathrm{dm}-\mathrm{cu}$ at least twice as long as $\mathrm{r}-\mathrm{m}$. Meron with unmodified setae. Legs yellowish brown, with femora distinctly darkened; hind tarsus with long, strong and dense setae ventrolaterally. Abdomen ground color dark brown or black. Male cercus short and broad, length/width ratio equal or less than 1.0; surstylus yellow, with an abruptly swollen lobe near base and a rounded apex; surstylar setae short, reaching at 


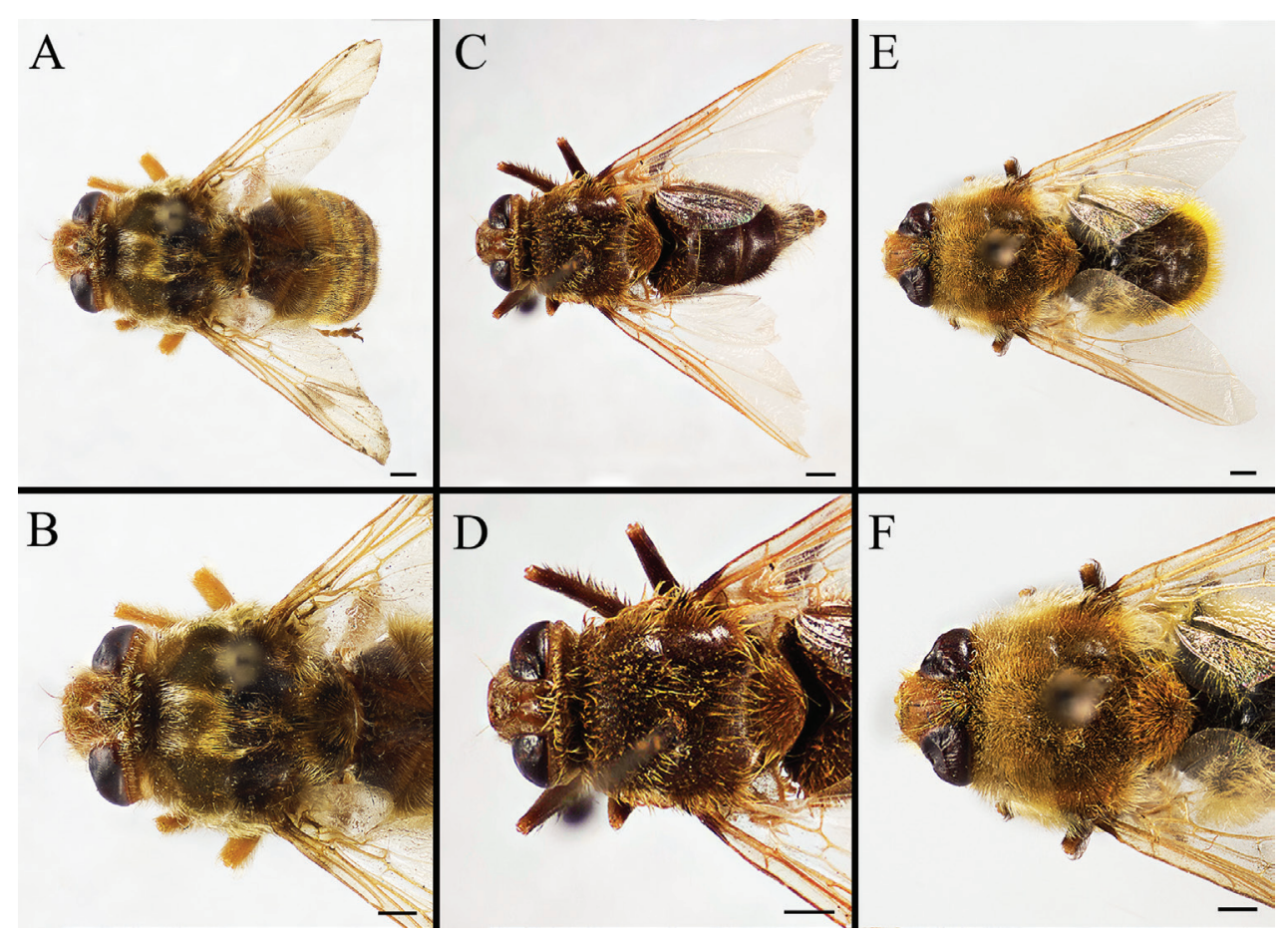

Figure 5. Dorsal view of habitus $(\mathbf{A}, \mathbf{C}, \mathbf{E})$ and head and and thorax $(\mathbf{B}, \mathbf{D}, \mathbf{F})$ of male Gasterophilus species A, B Male G. intestinalis (De Geer) C, D Female G. meridionalis (Pillers \& Evans) E, F Male G. nasalis (Linnaeus). Scale bars: $1 \mathrm{~mm}(\mathbf{A}-\mathbf{F})$.

most halfway to the sagittal plane; processi longi tubercular. Female sternite 8 longitudinally ridged in the middle and with a scallop-shaped apex.

Material examined. CHINA - Inner Mongolia $・ 20 \AA \widehat{\jmath}, 11$ 우; Chifeng, Zhaowuda League, Right Banner; 20 May-17 Sep. 1960; collector unknown; IOZ $\bullet 1 \overbrace{}^{\Uparrow}$; Ulanqab; Temurtei; 5 Jun. 1971; collector unknown; IOZ $\bullet 1$; ; Xilingol League, Dongwu Banner; 24 Aug. 1971; collector unknown; IOZ. - Heilongjiang Prov. • 10 ; Anda; 26 Jul. 1965; collector unknown; IOZ $\bullet 1$; Qiqihar; Fuyu County; 8 Aug. 1966; collector unknown; IOZ. - Xinjiang Uyghur Autonomous Region 10ิ; Wusu; 2000 m; 11 Jun. 1971; IOZ・1\%; Kalamaili; 3 Apr. 2011; D. Zhang leg.; MBFU・1 $\sigma^{\lambda}$; Kalamaili; 6 May 2011; D. Zhang leg.; MBFU.

Hosts. Burchell's zebra (E. quagga burchellii), domestic horse (E. ferus caballus Linnaeus), donkey (E. africanus asinus), Mongolian wild ass (E. hemionus hemionus Pallas), Mountain zebra (E. zebra Linnaeus), wild horse (E. przewalskii Poliakov).

Distribution. Afrotropical - Burkina Faso, Democratic Republic of the Congo, Eritrea, Ethiopia, Kenya, Namibia, Republic of the Congo, Senegal, South Africa, Sudan, Tanzania, Zambia. Australasian - Australia (New South Wales, Queensland, Victoria), Hawaii, New Zealand, Tasmania. Nearctic - Canada (Alberta, British Columbia, Manitoba, Saskatchewan), Mexico (no further data), USA (Colorado, Idaho, Illinois, Iowa, Kansas, Minnesota, Missouri, Montana, Nebraska, North Dakota, Or- 


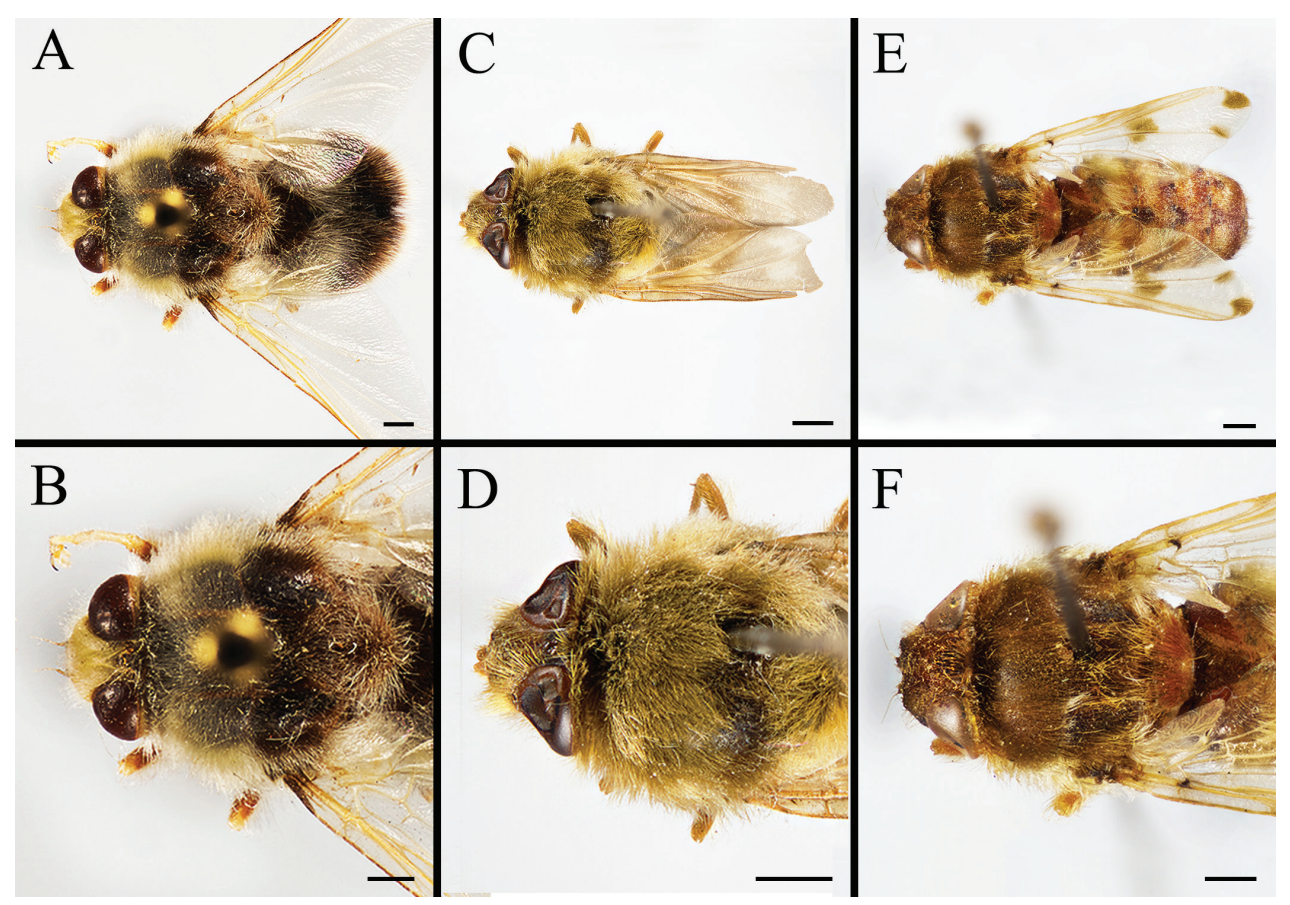

Figure 6. Dorsal view of habitus $(\mathbf{A}, \mathbf{C}, \mathbf{E})$ and head and thorax $(\mathbf{B}, \mathbf{D}, \mathbf{F})$ of male Gasterophilus species A, B G. nigricornis (Loew) C, D G. pecorum (Fabricius) E, F G. ternicinctus Gedoelst. Scale bars: $1 \mathrm{~mm}(\mathbf{A}-\mathbf{F})$.

egon, South Dakota, Utah, Virginia, Washington, Wisconsin, Wyoming). Neotropical - Argentina (no further data), Venezuela. Oriental - India. Palaearctic - Austria, Azerbaijan, Belgium, Bulgaria, China (Heilongjiang, Inner Mongolia, Qinghai, Shaanxi, Tibet, Xinjiang), Czech Republic, Denmark, Finland, France (incl. Corsica), Germany, Hungary, Iran, Iraq, Italy, Kazakhstan, Kyrgyzstan, Lithuania, Malta, Mongolia, Morocco, Palestine, Poland, Romania, Russia (Tomsk, Transbaikal, Yakutsk, Yenisseisk), Slovak Republic, Sweden, Switzerland, Tajikistan, The Netherlands, Turkey, Turkmenistan, Ukraine, United Kingdom, Uzbekistan.

\section{Gasterophilus inermis (Braurer, 1858)}

Figs 1G-I, 4E, F, 7G-I, 10C, 11G-I, 14G-I, 17I-L; Table 1

Gastrus inermis Brauer, 1858: 464. Type locality: Austria, Neusiedlersee, Jois (as "auf der Rossweide bei Gyois am Neusiedlersee").

Selected references. Brauer (1863: 73); Zumpt (1965: 124); Grunin (1969: 44); Soós and Mináŕ (1986: 238); Cogley (1991b); Xue and Wang (1996: 2209); Otranto et al. (2005); Colwell et al. (2006: 36); Li et al. (2018, 2019); Yan et al. (2019).

Diagnosis. Facial plate bare. Wing partly infuscate, with darkened patches with ill-defined edges. Distance between crossveins $\mathrm{r}-\mathrm{m}$ and $\mathrm{dm}$-cu less than length of $\mathrm{r}-\mathrm{m}$. 


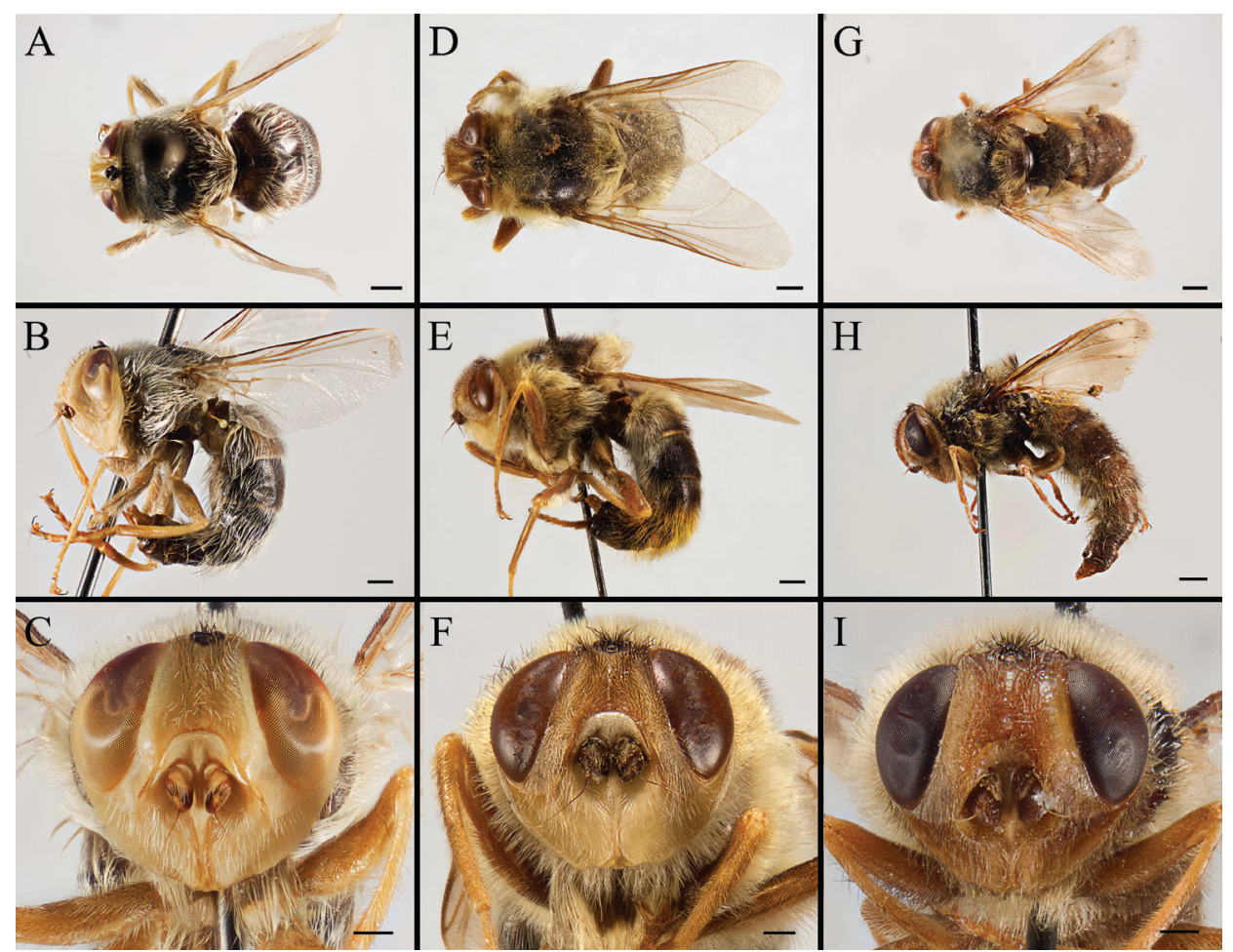

Figure 7. Dorsal view $(\mathbf{A}, \mathbf{D}, \mathbf{G})$ and left lateral view $(\mathbf{B}, \mathbf{E}, \mathbf{H})$ of habitus, and head in frontal view (C, F, I) of female Gasterophilus species, modified from Li et al. (2019) A-C G. flavipes (Olivier); China (in MBFU) D-F G. haemorrhoidalis (Linnaeus); China (in MBFU) G-I G. inermis (Brauer); Germany (in NHMD). Scale bars: $1 \mathrm{~mm}(\mathbf{A}, \mathbf{B}, \mathbf{D}, \mathbf{E}, \mathbf{G}, \mathbf{H}) ; 0.5 \mathrm{~mm}(\mathbf{C}, \mathbf{F}, \mathbf{I})$.

Meron bare. Legs yellowish brown, with femora distinctly darkened. Abdomen ground color yellow. Male cercus short and broad, length/width ratio equal or less than 1.0; surstylus yellow, with a rounded apex; processi longi tubercular. Female sternite 8 longitudinally ridged in the middle and with a scallop-shaped apex.

Type material examined. Syntypes of Gastrus inermis Brauer, 1858. AUSTRIA • $1{ }^{\lambda}, 1$; $;$ no further data; NHMW [from photo].

Additional material examined. AUSTRIA $\bullet 1$; ; no locality data; 31 Jul. 1986; Waldegg leg.; NHMW [from photo] $\bullet 1 \delta^{\lambda}$; 1892; no further data; NHMW [from photo]. ROMANIA • 1ठ, 1우 G. Dinulescu leg.; no further data; MNHN. GERMANY $\bullet 1 \hat{\jmath}, 1$; ; 1918; Wüstnei leg.; no further data; NHMD. CHINA - Inner Mongolia • 10ึ; Chifeng, Zhaowuda League, Right Banner; 16 Aug. 1969; collector unknown; IOZ $\bullet 1$; ; locality as for preceding; 22 Aug. 1969; IOZ.

Hosts. Burchell's zebra (E. quagga burchellii), domestic horse (E. ferus caballus), Mongolian wild ass (E. hemionus hemionus), wild horse (E. przewalskii).

Distribution. Afrotropical - Senegal, South Africa. Nearctic - USA (Illinois). Palaearctic - Austria, China (Inner Mongolia, Xinjiang), Germany, Hungary, Iran, Italy, Kazakhstan, Kyrgyzstan, Romania, Mongolia, Moldova, Slovak Republic, Tajikistan, Turkmenistan, Ukraine, Uzbekistan. 
Remarks. Brauer (1858: 465) explicitly states that he examined "one pair" of adults that were hatched from puparia collected by the Austrian entomologist Alois Friedrich Rogenhofer in horse dung. $1 \widehat{\delta}, 1 q$ in NHMW each carry two labels with the information "Oesterreich / Coll. Brauer" and "inermis / det Brauer". A fragment of a puparium carries labels with "Gastrus / inermis / det Brauer" and "Coll. Brauer". We consider the pair of adults to most probably represent original syntypes, but we are deliberately abstaining from designating a lectotype at this time.

\section{Gasterophilus intestinalis (De Geer, 1776)}

Figs 2A-C, 5A, B, 8A-C, 10D, 12A-C, 15A-C, 17M-P; Table 1

Oestrus intestinalis De Geer, 1776: 292. Type locality: Sweden.

Oestrus equi Clark, 1797: 298. Junior primary homonym of Oestrus equi Fabricius, 1787. Type locality: England.

Oestrus gastricus major Schwab, 1840: 31. Unavailable name; proposed in synonymy with Oestrus intestinalis De Geer, 1776 and Oestrus equi Clark, 1797 and not made available from subsequent use as a valid name for a taxon before 1961.

Oestrus bengalensis Macquart, 1843: 182. Type locality: Bangladesh (as "Du Bengal") and India.

Oestrus gastrophilus Gistel, 1848: 153 (as “O. gastrophilus, mihi. O. Equi. Linné.”). Type locality: not given, probably Germany.

Oestrus schwabianus Gistel, 1848: 153 (as "Oestrus Schwabianus, mihi. O. gastric. major Schwab”). Type locality: not given, probably Germany, Bavaria.

Gastrophilus equi var. asininus Brauer, 1863: 71. Type locality: Egypt and Sudan (“Egypten" \& "Nubien").

Gastrophilus aequi: Brauer 1863: 28; incorrect subsequent spelling of equi Clark, 1797. Gasterophilus magnicornis Bezzi, 1916: 29. Type locality: Eritrea.

Selected references. Zumpt (1965: 125); Grunin (1969: 48); Pont (1973: 698); James (1974: 96); Kettle (1974); Soós and Mináŕ (1986: 238); Cogley (1991b); Escartin and Bautista (1993); Xue and Wang (1996: 2210); Otranto et al. (2005); Colwell et al. (2006: 4); Colwell et al. (2007); Felix et al. (2007); Güiris et al. (2010); Ganjali and Keighobadi (2016); Zhang et al. (2016); Li et al. (2018); Yan et al. (2019).

Diagnosis. Facial plate bare. Wing partly infuscate, with darkened patches with ill-defined edges; crossvein dm-cu situated almost opposite of crossvein r-m. Meron with unmodified setae. Legs yellow, with more or less dark coloration on tarsus; hind trochanter with a spatulate process in male and a tubercle in female. Abdomen ground color yellow in both male and female. Male cercus elongated and broad, length-width ratio around 1.5; surstylus mainly yellow with black coloration apically, and a rounded apex; processi longi elongated. Female abdominal segment 7 distinctly longer than broad, sternite 8 longitudinally ridged in the middle and with a scallop-shaped apex. 


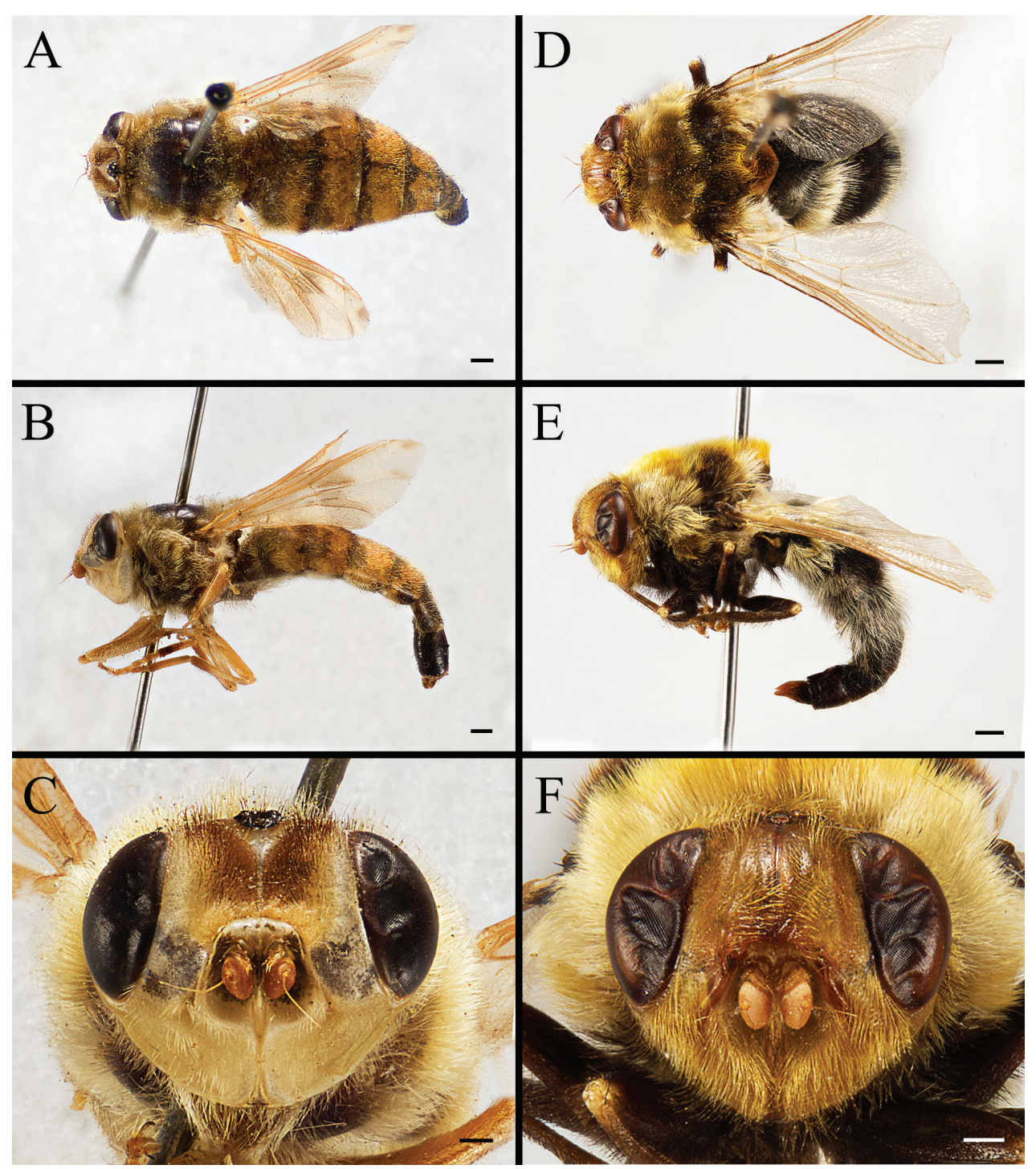

Figure 8. Dorsal view $(\mathbf{A}, \mathbf{D}, \mathbf{G})$ and left lateral view $(\mathbf{B}, \mathbf{E}, \mathbf{H})$ of habitus, and head in frontal view $(\mathbf{C}, \mathbf{F}, \mathbf{I})$ of female Gasterophilus species A-C G. intestinalis (De Geer); China (in MBFU) D-F G. nasalis (Linnaeus); China (in MBFU). Scale bars: $1 \mathrm{~mm}(\mathbf{A}, \mathbf{B}, \mathbf{D}, \mathbf{E}) ; 0.5 \mathrm{~mm}(\mathbf{C}, \mathbf{F})$.

Material examined. CHINA •- Inner Mongolia ・13 $\widehat{\partial}, 2690$; Chifeng; Zhaowuda League, Right Banner; 13 Jun.-17 Sep. 1960; collector unknown; IOZ $\bullet 1$; Hulunbeir; Genhe; 13 Aug. 1971; collector unknown; IOZ •19; Hulunbeir; Yakeshi; 19 Aug. 1971; collector unknown; IOZ $\bullet 1$; ; Hailaer; 23 Aug. 1971; collector unknown; IOZ - 19; Hulunbeir; Yakeshi; Boketu; 28 Aug 1971; collector unknown;

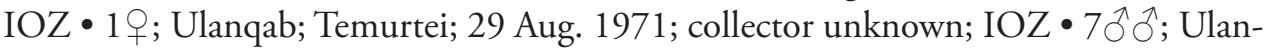




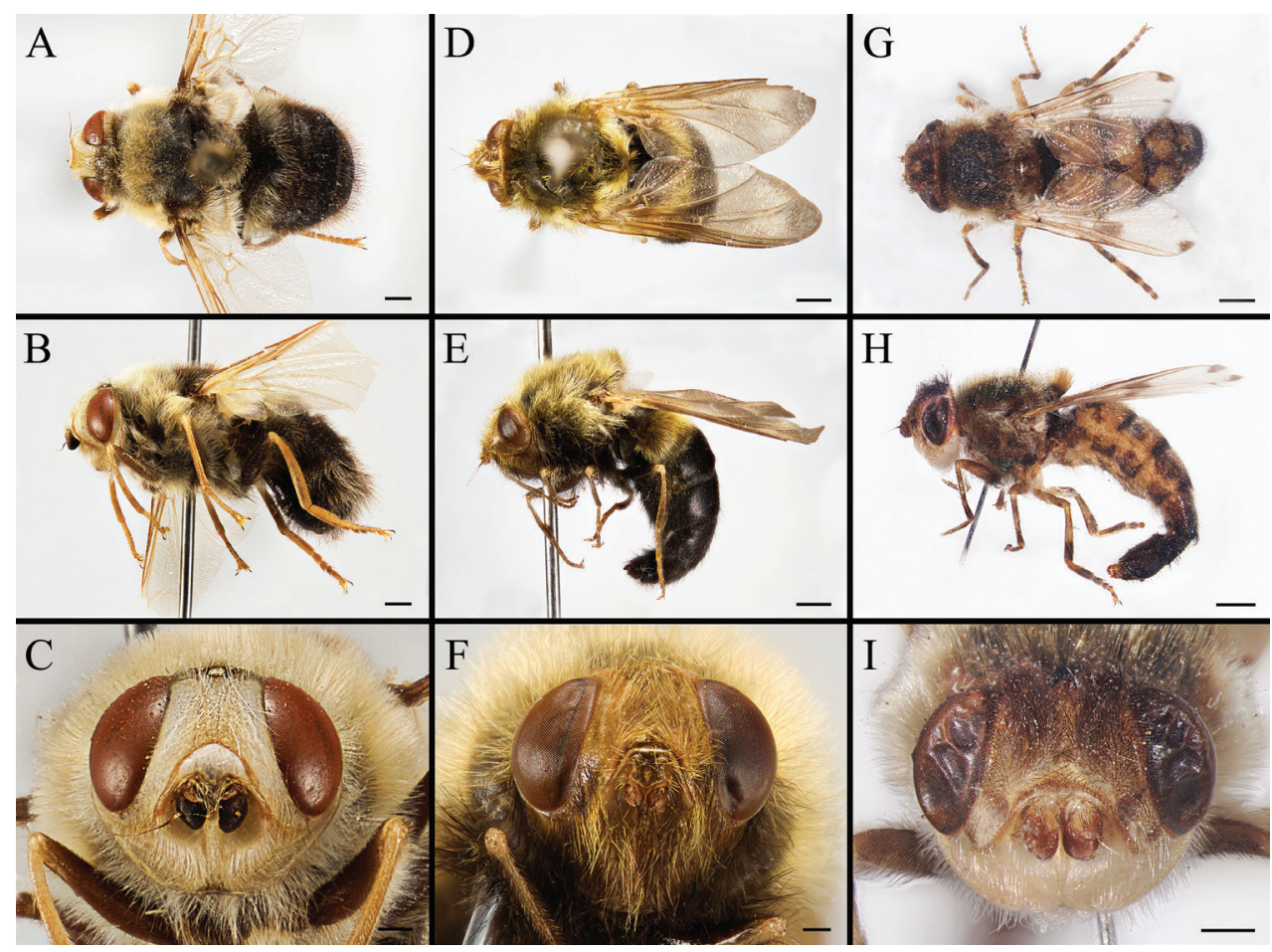

Figure 9. Dorsal view $(\mathbf{A}, \mathbf{D}, \mathbf{G})$ and left lateral view $(\mathbf{B}, \mathbf{E}, \mathbf{H})$ of habitus, and head in frontal view (C, F, I) of female Gasterophilus species A-C G. nigricornis (Loew); China (in MBFU) D-F G. pecorum (Fabricius); China (in MBFU) G-I G. ternicinctus Gedoelst; Kenya (in NHM). Scale bars: $1 \mathrm{~mm}(\mathbf{A}, \mathbf{B}$, D-E, G, H); $0.5 \mathrm{~mm}(\mathbf{C}, \mathbf{F}, \mathbf{I})$.

qab; Temurtei; 29 Aug. 1971; collector unknown; IOZ $\bullet 2$ 2q; Ulanqab, Temurtei; 30 Aug. 1971; collector unknown; IOZ. - Heilongjiang Prov. $\bullet 2 \widehat{\jmath}, 2$, 9 ; Anda; 26-27 Aug. 1965; collector unknown; IOZ $\bullet 3$ 우; Qiqihar, Fuyu County; 15 Jun.26 Aug. 1966; collector unknown; IOZ $\bullet 1$; Daqing, Lamadian County; 15 Aug. 1969; collector unknown; IOZ $\bullet 1$; ; locality as for preceding; 17 Sep. 1969; collector unknown; IOZ $\bullet 1$; ; Qiqihar, Tailai County, Jiangning; 20 Jun. 1970; collector

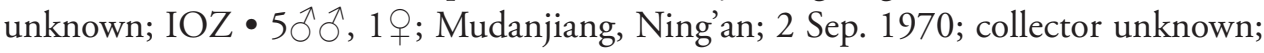
IOZ. - Beijing • 19; Yanqing County; 4 Aug. 1970; collector unknown; IOZ. - Tibet Autonomous Region - 1ठ; Xinglin; 2550 m; 18 Aug. 1974; collector unknown; IOZ. - Sichuan Prov. •19; Aba Autonomous Prefecture, Hongyuan County; 3700 m; 27 Aug. 1983; collector unknown; IOZ $\bullet 1$; ; locality as for preceding; 3500 m; 28

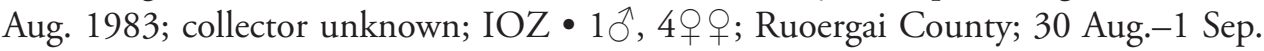
1983; collector unknown; IOZ. 1 1 ; no further data; MNHN.

Hosts. Domestic horse (E. ferus caballus), donkey (E. africanus asinus), Mongolian wild ass (E. hemionus hemionus), wild horse (E. przewalskii).

Distribution. Afrotropical - Burkina Faso, Chad, Eritrea, Ethiopia, Ghana, Kenya, Morocco, Nigeria, Republic of the Congo, Senegal, South Africa, Sudan, Tanza- 


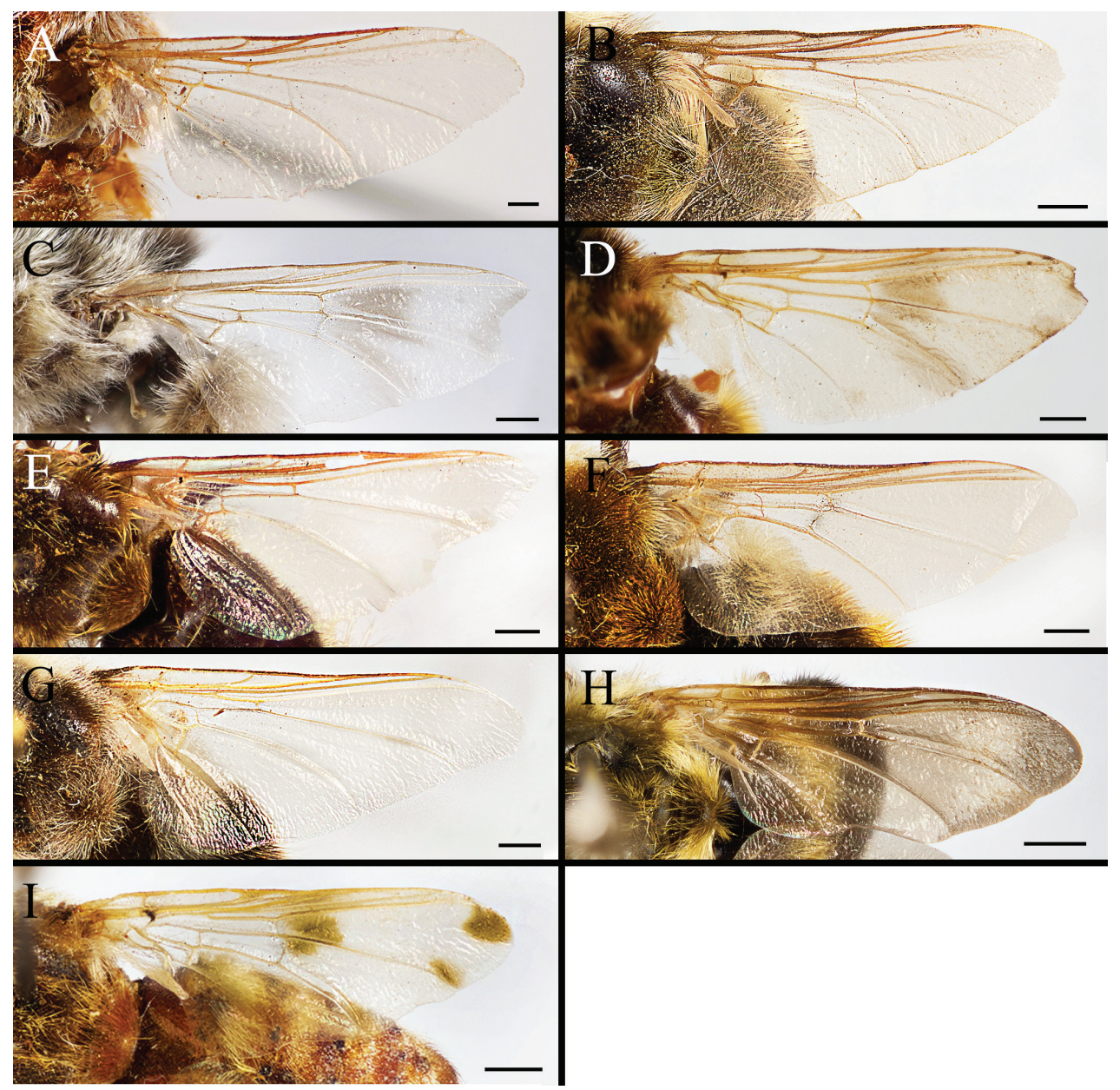

Figure 10. Wing of Gasterophilus species, with A-C modified from Li et al. (2019) A G. flavipes (Olivier) B $G$. haemorrhoidalis (Linnaeus) C $G$. inermis (Brauer) D G. intestinalis E G. meridionalis (Pillers \& Evans) $\mathbf{F}$ G. nasalis (Linnaeus) G G. nigricornis (Loew) H G. pecorum (Fabricius) I G. ternicinctus Gedoelst. Scale bars: $0.5 \mathrm{~mm}(\mathbf{A}-\mathbf{C}) ; 1 \mathrm{~mm}(\mathbf{D}-\mathbf{I})$.

nia. Australasian - Australia (New South Wales, Norfolk I, Tasmania), Hawaii, New Zealand. Nearctic - Canada (Alberta, British Columbia, Manitoba, New Brunswick, Ontario, Quebec, Saskatchewan), Mexico (Aguascalientes, Chiapas), USA (Arizona, California, Colorado, Connecticut, Idaho, Illinois, Iowa, Kansas, Maine, Maryland, Massachusetts, Michigan, Minnesota, Mississippi, Missouri, Montana, Nebraska, New Hampshire, New Jersey, New Mexico, New York, North Carolina, North Dakota, Ohio, Oklahoma, Oregon, South Dakota, Texas, Utah, Vermont, Virginia, Washington, Wisconsin, Wyoming). Neotropical - Argentina (no further data), Brazil (Rio Grande do Sul), Chile (Bío Bío Region), Jamaica, Venezuela. Oriental - India. Palaearctic - Bangladesh, Belgium, China (Beijing, Gansu, Heilongjiang, Inner Mongolia, Qinghai, Shanxi, Shaanxi, Sichuan, Tibet, Xinjiang, Yunnan), Czech 
Republic, Denmark, Egypt, Finland, France (incl. Corsica), Germany, Hungary, Ireland, Iran, Italy (incl. Sicily), Jordan, Lithuania, Mongolia, Norway, Pakistan, Poland, Romania, Slovak Republic, Sweden, Switzerland, The Netherlands, Turkey, Ukraine, United Kingdom.

Gasterophilus meridionalis (Pillers \& Evans, 1926)

Figs 2D-F, 5C, D, 11E, 15D-F; Table 1

Oestrus meridionalis Pillers \& Evans, 1926: 264. Type locality: Zimbabwe (as “Rhodesia”).

Selected references. Zumpt (1965: 121); Cogley (1991b); Colwell et al. (2006: 36); Colwell et al. (2007: 256).

Diagnosis. Male unknown. Antennal postpedicel long-oval. Facial plate setose. Wing completely hyaline. Crossvein $\mathrm{dm}$-cu extremely weak, with only a faint trace; distance between crossveins $\mathrm{r}-\mathrm{m}$ and $\mathrm{dm}-\mathrm{cu}$ equal or less than length of $\mathrm{r}-\mathrm{m}$. Meron with unmodified setae. Legs black or black-brown. Abdomen ground color dark brown. Female sternite 8 longitudinally ridged in the middle and with a scallop-shaped apex.

Material examined. SOUTH AFRICA - 2 \% ; Transvaal; Newington; 15 Aug. 1957; reared from third instar larvae by F. Zumpt; KZNM.

Hosts. Burchell's zebra (E. quagga burchellii).

Distribution. Afrotropical - Botswana, Democratic Republic of the Congo, Mozambique, Namibia, Republic of the Congo, South Africa, Tanzania, Zambia, Zimbabwe.

\section{Gasterophilus nasalis (Linnaeus, 1758)}

Figs 2G-I, 5E, F, 8D-F, 10F, 11F, 12D-F, 15G-I; Table 1

Oestrus nasalis Linnaeus, 1758: 584. Type locality: Sweden (through reference to Fauna Svecica).

Oestrus equi Fabricius, 1787: 321. Type locality: not given, probably Europe.

Oestrus veterinus Clark, 1797: 312. New replacement name for Oestrus nasalis Linnaeus, 1758 ["I have given it the name of veterinus .... in preference to the erroneous one of nasalis" (p. 313)].

Oestrus salutaris Clark, 1815: pl. 1. Nomen nudum.

Gasterophilus clarkii Leach, 1817: 2. Type locality: England, Bantham close to Kingsbridge (as "Habitat in Anglia Occidentali. Apud Bantham prope Kingsbridge a meipso captus").

Gastrus jumentarum Meigen, 1824: 179. Type locality: not given, probably Denmark (as "Ein Weibchen in dem Koppenhagener Königl. Museum").

Oestrus gastricus minor Schwab, 1840: 40. Unavailable name proposed in synonymy with Oestrus nasalis Linnaeus, 1758 and Oestrus veterinus Clark, 1797 and not made available from subsequent use as a valid name for a taxon before 1961 . 


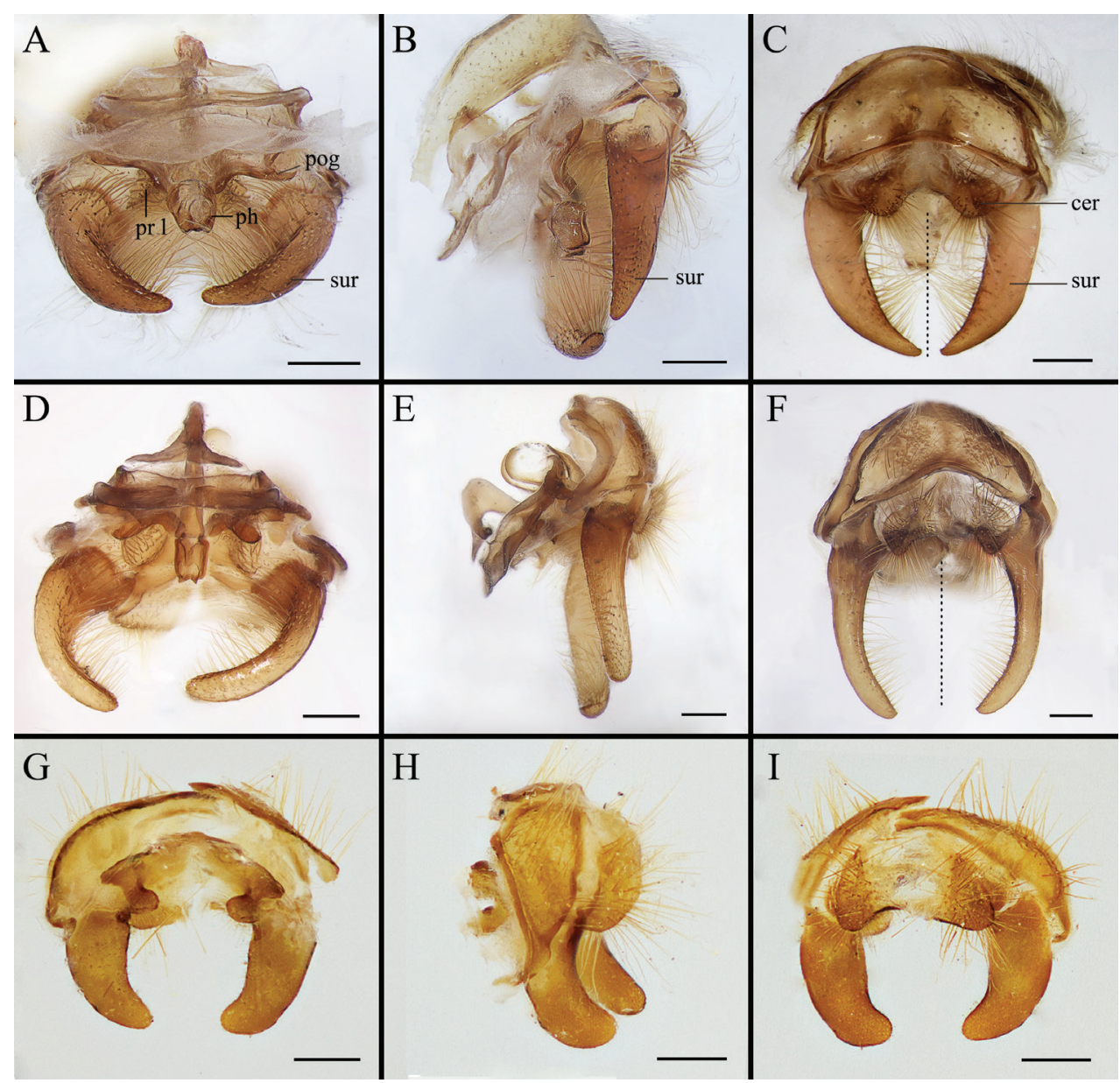

Figure II. Anterior (A, D, G), left lateral (B, E, H) and posterior view $(\mathbf{C}, \mathbf{F}, \mathbf{I})$ of male terminalia of Gasterophilus species, modified from Li et al. (2019) A-C G. flavipes (Olivier) D-F G. haemorrhoidalis (Linnaeus) G-I G. inermis (Brauer). Scale bars: $0.5 \mathrm{~mm}(\mathbf{A}-\mathbf{I})$. The dotted line in $\mathbf{C}$ and $\mathbf{F}$ indicates the sagittal plane. Abbreviations: cer, cercus; ph, phallus; pog, postgonite; pr l, processi longi; sur, sustylus.

Gastrus subjacens Walker, 1849: 687. Type locality: Canada, Nova Scotia.

Oestrus stomachinus Gistel, 1848: 153. Type locality: not given, probably Germany, Bavaria. Gasterophilus crossi Patton, 1924: 963. Type locality: India, Punjab.

Gastrophilus albescens Pleske, 1926: 228. Type locality: Egypt, Cairo (as "Il provient de l'Egypte des environs du Caire").

Gastrophilus nasalis var. nudicollis Dinulescu, 1932: 28, 32. Type locality: not given. Gastrophilus veterinus var. aureus Dinulescu, 1938: 315. Type locality: not given. Gastrus jumentorum: Brauer 1863: 87, 280; incorrect subsequent spelling of jumentarum Meigen, 1824.

Oestrus nasulis: Fabricius 1787: 321; incorrect subsequent spelling of nasalis Linnaeus, 1758. 


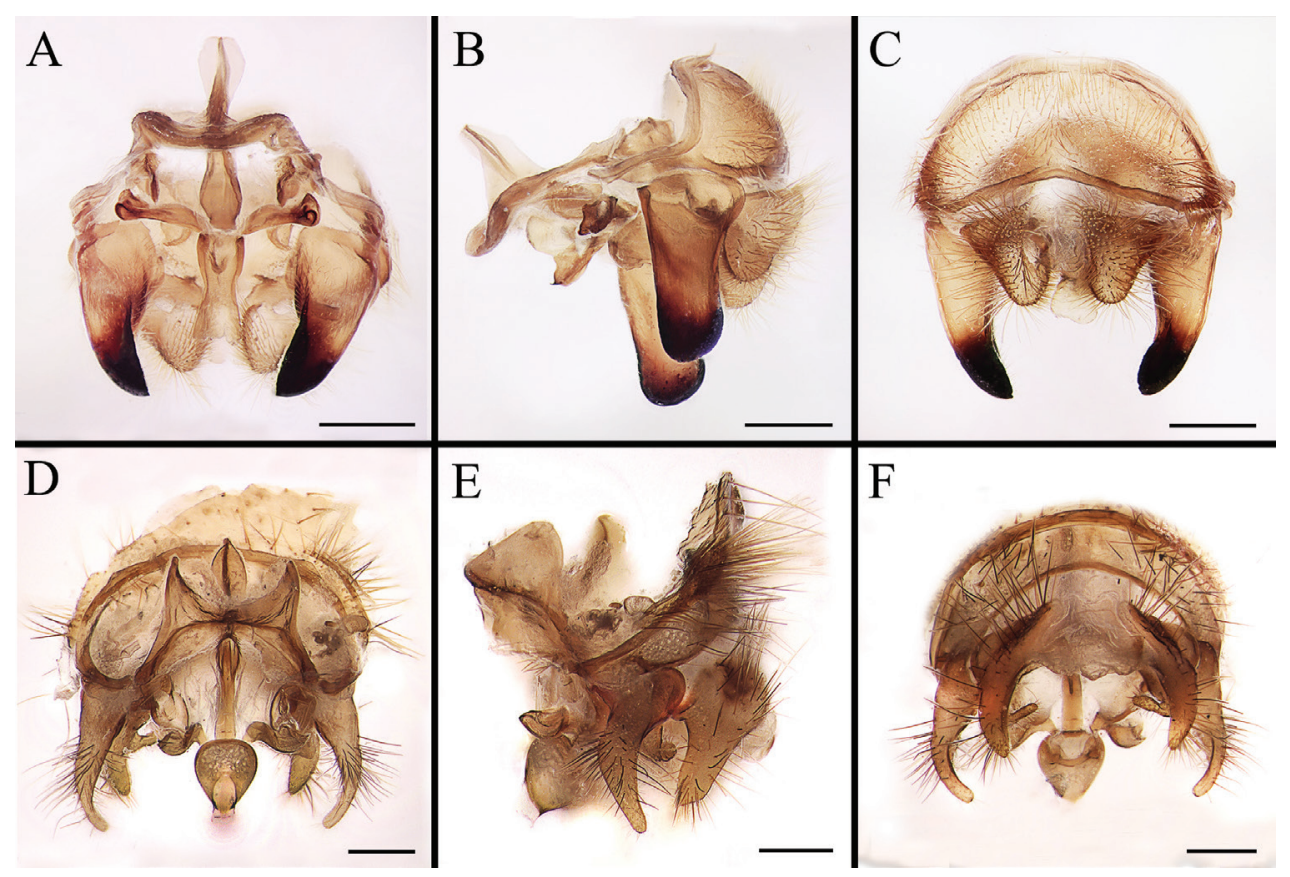

Figure I 2. Dorsal (A, D), left lateral (B, E) and posterior $(\mathbf{C}, \mathbf{F})$ view of male genitalia of Gasterophilus species A-C G. intestinalis (De Geer) D-F G. nasalis (Linnaeus). Scale bars: $0.5 \mathrm{~mm}(\mathbf{A}-\mathbf{F})$.

Selected references. Zumpt (1965: 117); Grunin (1969: 32); Pont (1973: 698); Kettle (1974); Soós and Mináŕ (1986: 238); Cogley (1991b); Escartin and Bautista (1993); Xue and Wang (1996: 2210); Sequeira et al. (2001); Otranto et al. (2005); Colwell et al. (2006: 6); Colwell et al. (2007); Felix et al. (2007); Zhang et al. (2016); Li et al. (2018); Yan et al. (2019).

Diagnosis. Facial plate setose. Wing entirely hyaline; distance between crossveins $\mathrm{r}-\mathrm{m}$ and $\mathrm{dm}-\mathrm{cu}$ less than length of $\mathrm{r}-\mathrm{m}$. Meron with unmodified setae. Legs mainly black-brown. Abdomen ground color dark brown or black, with reddish-yellow hairlike setae on tergites 5-7 in male, pale yellow in female. Male cercus long and narrow, length/width ratio more than 3.0; surstylus yellow, with gradually a tapered apex; processi longi elongated and distinctly bent inwards. Female sternite 8 longitudinally ridged in the middle and with flattened and a scallop-shaped apex.

Type material examined. Holotype of Gastrophilus albescens Pleske, 1926. EGYPT - $\jmath^{7}$; Cairo; no further information; ZIN.

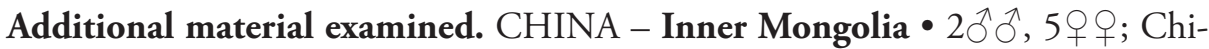
feng; Zhaowuda League, Right Banner; 24 May-10 Aug. 1960; collector unknown; IOZ • 5Ô, 1우 Ulanqab, Temurtei County; 12-30 Aug. 1971; Y.R. Zhang leg.; IOZ. - Xinjiang Uyghur Autonomous Region • 1今; Altay, Qinghe County; 6 Jul. 1960; S.Y. Wang leg.; IOZ • 1q; Altyn-Tagh; 3850 m; 7 Aug. 1988; X.Z. Zhang leg.; IOZ

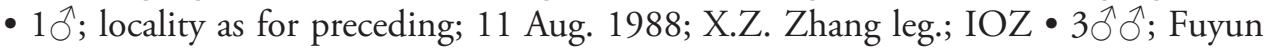
County; Qiakuertu; 25 May-3 Jun. 2010; F. Mo leg.; MBFU・8ふึત, 1 여 Kalamaili; 


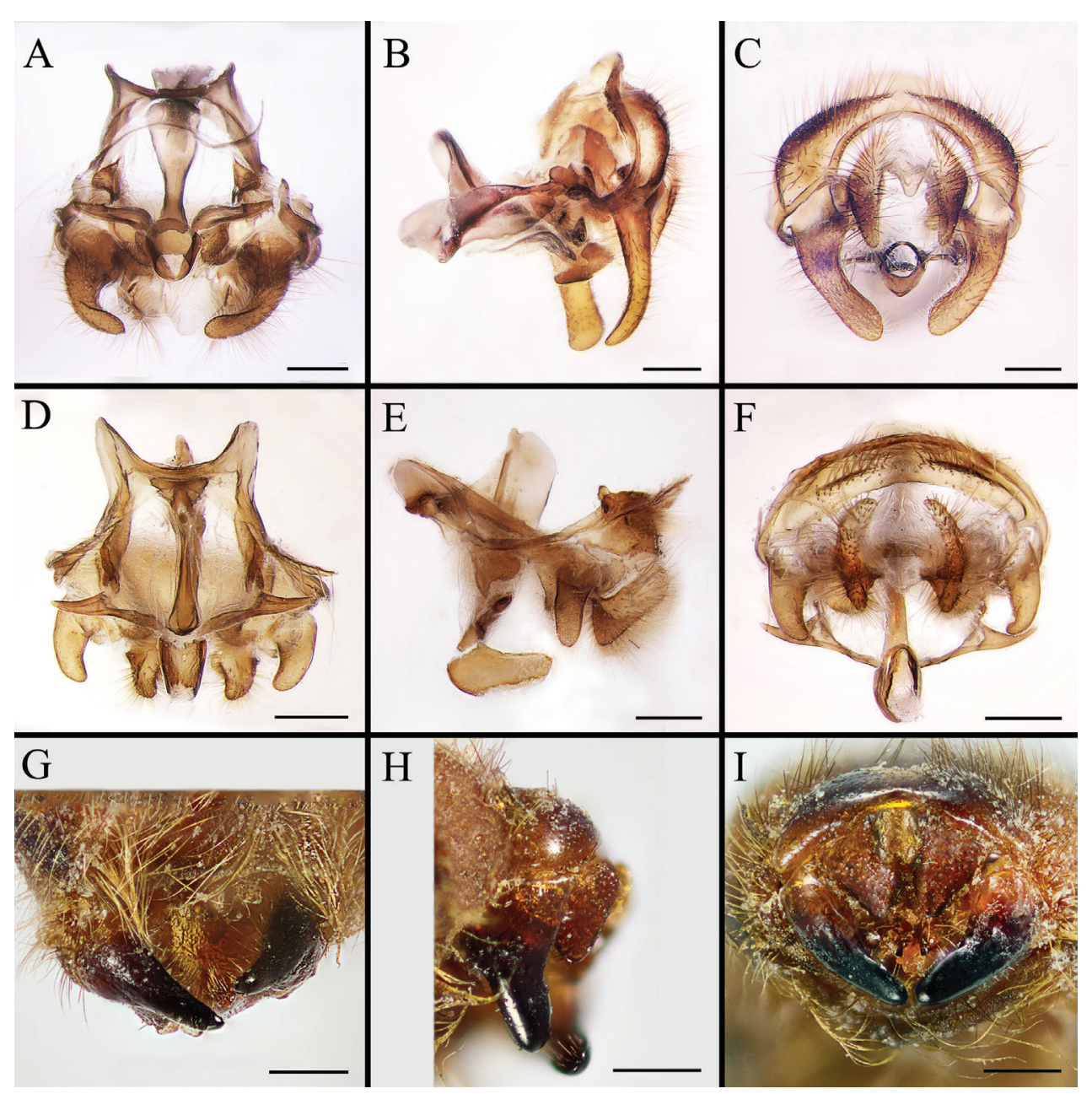

Figure 13. Dorsal (A, D, G), left lateral (B, E, H) and posterior $(\mathbf{C}, \mathbf{F}, \mathbf{I})$ view of male genitalia of Gasterophilus species A-C G. nigricornis (Loew) D-F G. pecorum (Fabricius) G-I G. ternicinctus Gedoelst. Scale bars: $0.5 \mathrm{~mm}(\mathbf{A}-\mathbf{I})$.

18 Apr.-25 Jun. 2010; D. Zhang leg.; MBFU・1ðð, 5 우; Kalamaili; 16 Apr.-8 May 2011; D. Zhang leg.; MBFU.

Hosts. Burchell's zebra (E. quagga burchellii), domestic horse (E. ferus caballus), donkey (E. africanus asinus), Mongolian wild ass (E. hemionus hemionus), wild horse (E. przewalskii).

Distribution. Afrotropical - Burkina Faso, Ethiopia, Kenya, Lesotho, Morocco, Namibia, Senegal, South Africa, Zambia, Zimbabwe. Australasian - Australia (Queensland, Tasmania), Fiji, Hawaii, New Zealand. Nearctic - Canada (Alberta, British Columbia, Manitoba, Northwestern, Nova Scotia, Quebec, Saskatchewan), Mexico (Aguascalientes, San Vicente Chicoloapan), USA (Arizona, California, Colorado, Illinois, Iowa, Kansas, Kentucky, Maryland, Michigan, Minnesota, Missouri, 


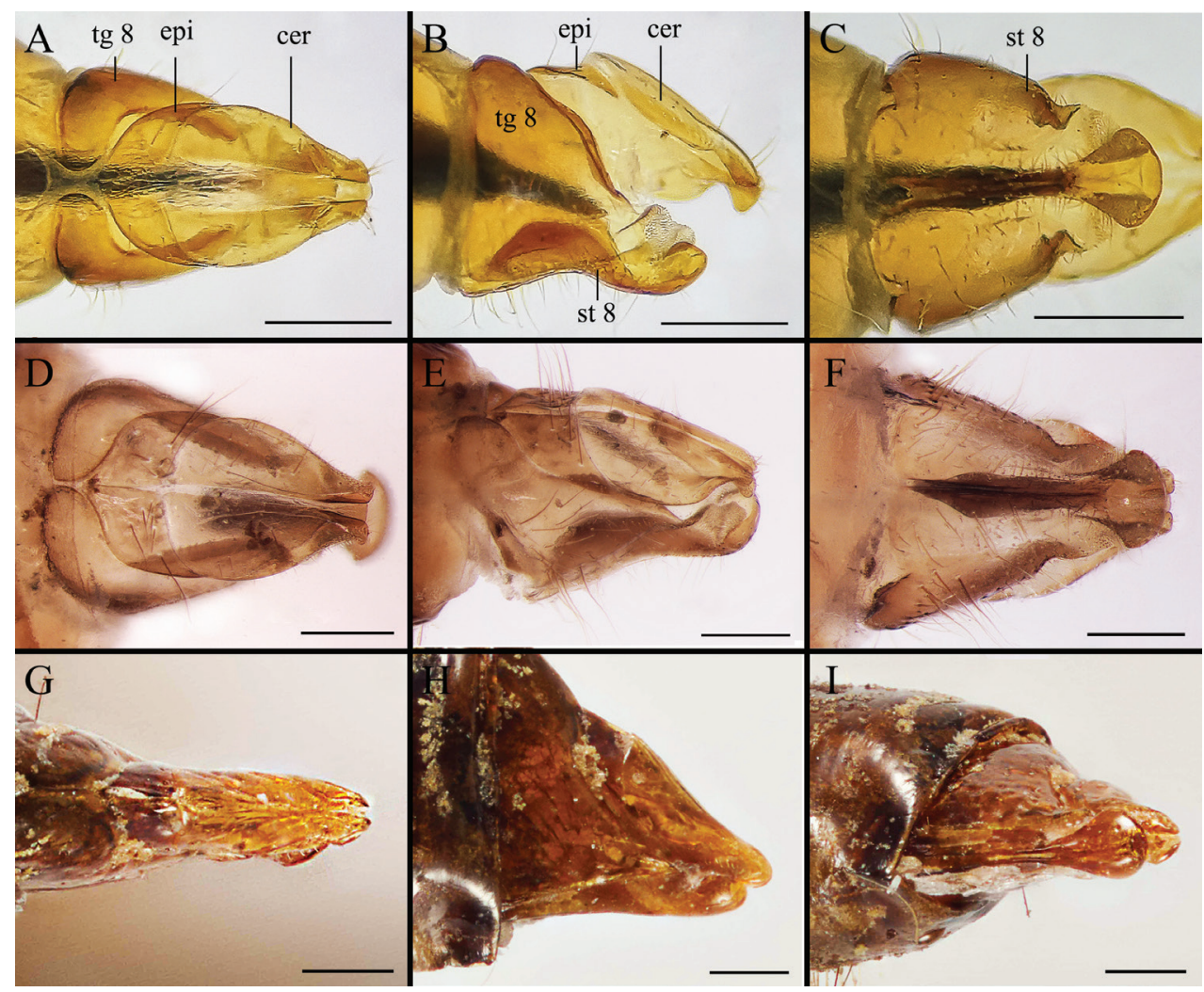

Figure 14. Dorsal (A, D, G), left lateral $(\mathbf{B}, \mathbf{E}, \mathbf{H})$ and ventral $(\mathbf{C}, \mathbf{F}, \mathbf{I})$ view of female genitalia of Gasterophilus species, modified from Li et al. (2019) A-C G. flavipes (Olivier) D-F G. haemorrhoidalis (Linnaeus) G-I G. inermis (Brauer). Scale bars: $0.5 \mathrm{~mm}(\mathbf{A}-\mathbf{C}) ; 1 \mathrm{~mm}$ (D-I). Abbreviations: cer, cercus; epi, epiproct; sg 7, segment 7; sl, stalk-like pedicel; st 8 , sternite 8 ; tg 8 , tergite 8 .

Montana, Nebraska, New Jersey, New Mexico, New York, North Dakota, Ohio, Oklahoma, Oregon, South Dakota, Texas, Washington, Wyoming). Neotropical - Antigua and Barbuda, Argentina, Brazil (Rio Grande do Sul, São Paulo), Chile (Bío Bío Region), Jamaica, Panama, Puerto Rico, Uruguay, Venezuela. Oriental - India, Malaysia, Myanmar, Thailand. Palaearctic - Afghanistan, Austria, Bulgaria, China (Heilongjiang, Inner Mongolia, Shaanxi, Tibet, Xinjiang), Cyprus, Denmark, Egypt, France, Germany, Hungary, Iraq, Italy (incl. Corsica and Sicily), Jordan, Kazakhstan, Kyrgyzstan, Lithuania, Mongolia, Morocco, Pakistan, Poland, Romania, Russia (Tomsk), Sweden, Switzerland, The Netherlands, Tajikistan, Turkey, Turkmenistan, Ukraine, United Kingdom, Uzbekistan.

\section{Gasterophilus nigricornis (Loew, 1863)}

Figs 3A-C, 6A, B, 9A-C, 10G, 13A-C, 16A-C, 18E-H; Table 1

Gastrus nigricornis Loew, 1863: 38. Type locality: Moldova, Bessarabia (as “Bessarabien”). 


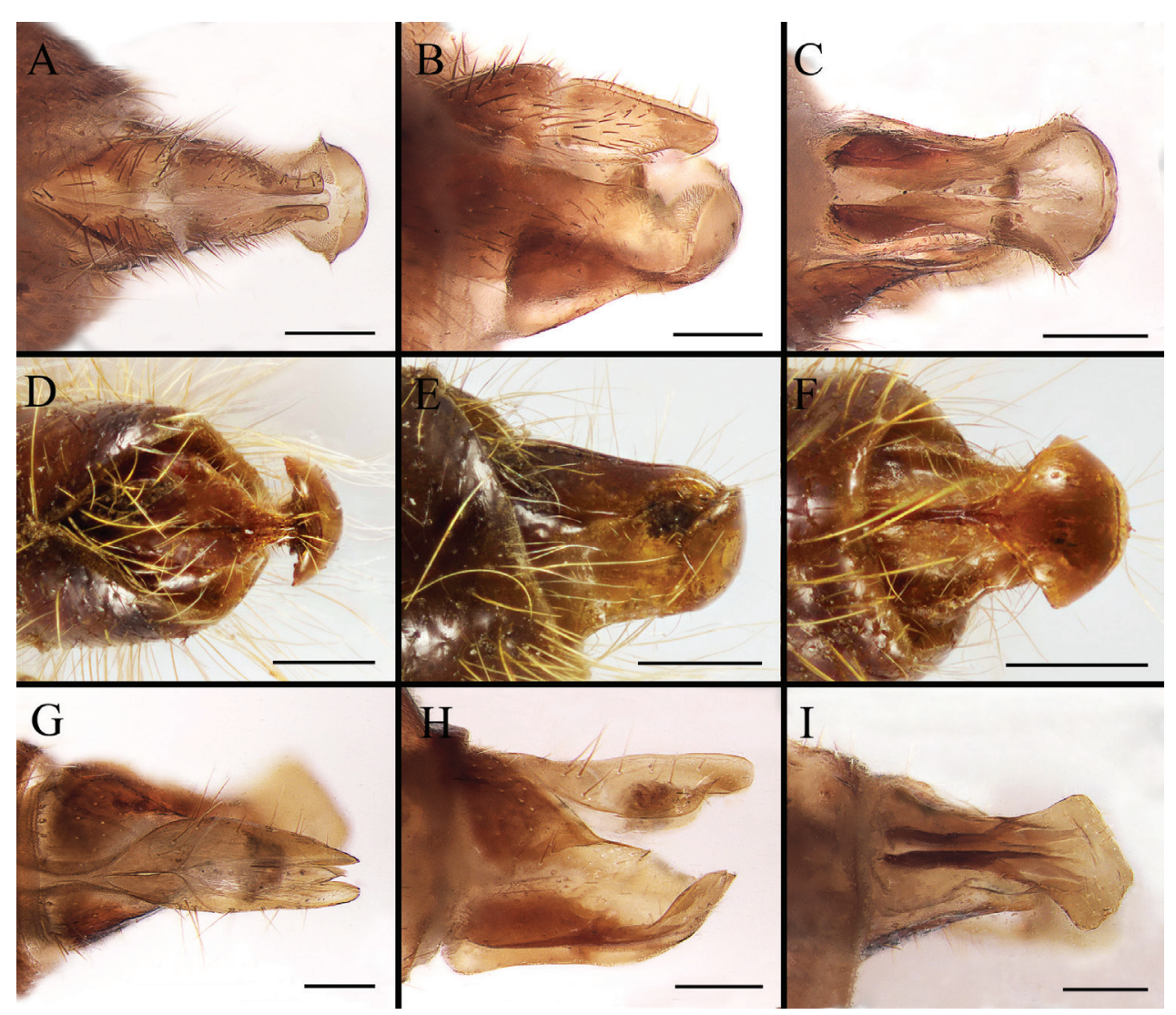

Figure 15. Dorsal (A, D, G), left lateral $(\mathbf{B}, \mathbf{E}, \mathbf{H})$ and ventral $(\mathbf{C}, \mathbf{F}, \mathbf{I})$ view of female genitalia of Gasterophilus species A-C G. intestinalis (De Geer) D-F G. meridionalis (Pillers \& Evans) G-I G. nasalis (Linnaeus). Scale bars: $0.5 \mathrm{~mm}(\mathbf{A}-\mathbf{I})$.

Gastrophilus viridis Sultanov, 1951: 41. Type locality: Kazakhstan, Kzyl-Ordinskaja, around Teren-Uzyakaskiy.

Gasterophilus migricornis: Colwell 2006: 291; incorrect subsequent spelling of nigricornis Loew, 1863.

Selected references. Zumpt (1965: 119); Grunin (1969: 36); Soós and Minár (1986: 239); Xue and Wang (1996: 2214); Colwell et al. (2006: 36); Zhang et al. (2012, 2016); Li et al. (2018); Yan et al. (2019).

Diagnosis. Antennal postpedicel red-brown to blackish. Facial plate setose. Meral setae with swollen tip. Wing completely hyaline. Crossvein dm-cu absent. Legs yellowish brown with femora distinctly darkened. Male cercus long and narrow, length/width ratio more than 3.0; surstylus yellow, with a rounded apex; processi longi elongated. Female sternite 8 longitudinally ridged in the middle and with a scallop-shaped apex.

Material examined. CHINA - Xinjiang Uyghur Autonomous Region ・ 1ð; Barköl Kazak Autonomous County, Saerqiaoke; 14 Aug. 1968; collector unknown; 


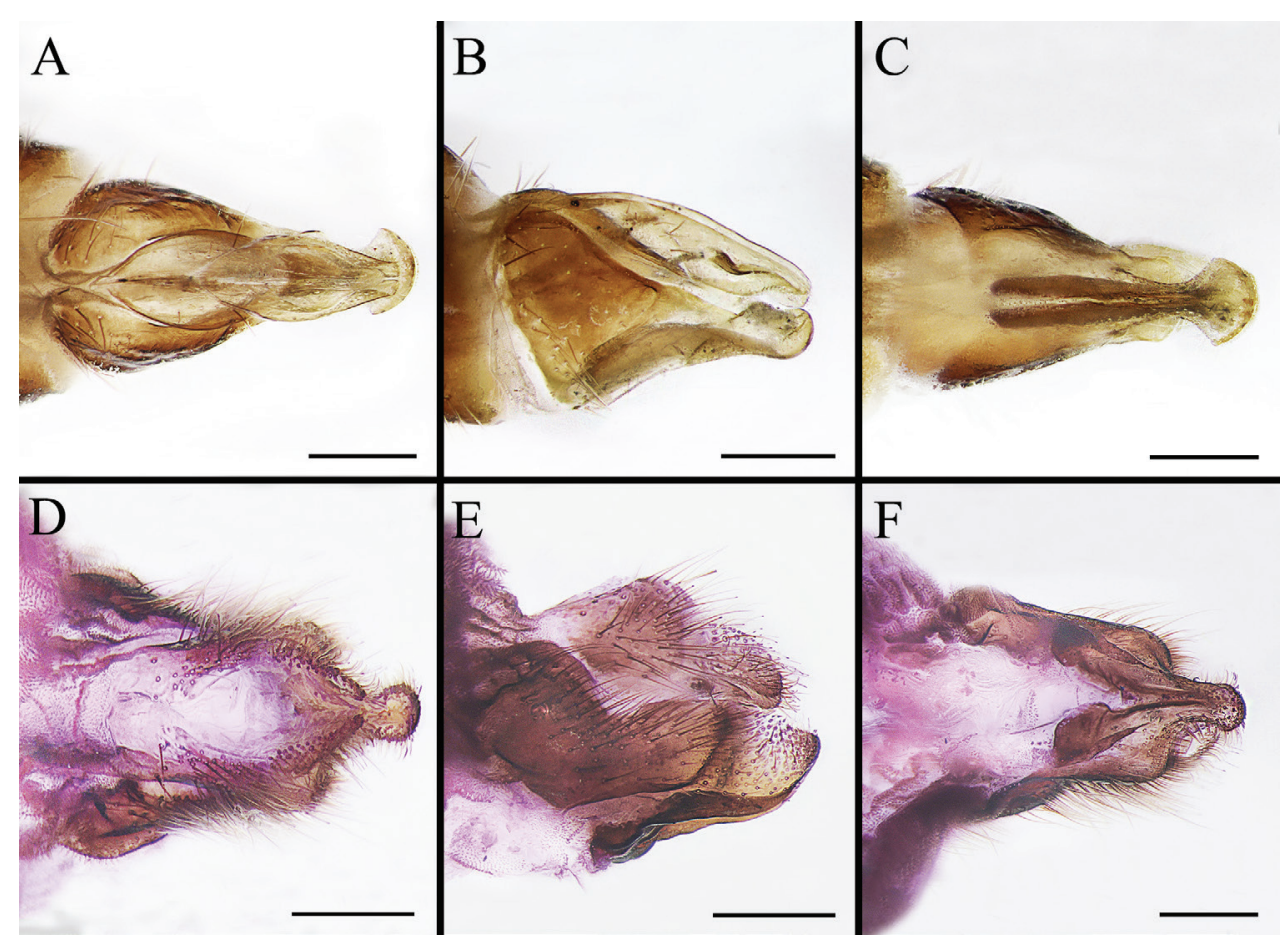

Figure 16. Dorsal (A, D), left lateral (B, E) and ventral $(\mathbf{C}, \mathbf{F})$ view of female genitalia of Gasterophilus species A-C G. nigricornis (Loew) D-F G. pecorum (Fabricius). Scale bars: $0.5 \mathrm{~mm}(\mathbf{A}-\mathbf{F})$.

IOZ・9ðえ, 19; Kalamaili; 3 Apr.-18 May 2009; D. Zhang leg.; MBFU・27ðð, 19; Fuyun County, Qiakuertu; 25 Apr.-13 May 2009; F. Mo leg.; MBFU.

Hosts. Domestic horse (E. ferus caballus), donkey (E. africanus asinus), Mongolian wild ass (E. hemionus hemionus), wild horse (E. przewalskii).

Distribution. Palaearctic - China (Inner Mongolia, Qinghai, Xinjiang), Kazakhstan, Kyrgyzstan, Moldova, Mongolia, Tajikistan, Turkmenistan, Ukraine, Uzbekistan.

Remarks. The distribution of G. nigricornis appears to be limited to far eastern Europe and Central Asia. Thus, reports of G. nigricornis from western part of Europe [Spain: Lucientes (2002); Italy: Pape (2013)] are suspected to be misidentifications and the records are not included.

\section{Gasterophilus pecorum (Fabricius, 1794)}

Figs 3D-F, 6C, D, 9D-F, 10H, 13D-F, 16D-F, 18I-L; Table 1

Oestrus pecorum Fabricius, 1794: 230. Type locality: not given, probably Europe.

Oestrus vituli Fabricius, 1794: 231. Type locality: not given, but at least Sweden and

France by reference to works of Linnaeus and Geoffroy.

Gastrus jubarum Meigen, 1824: 179, 180. Type locality: Austria. 


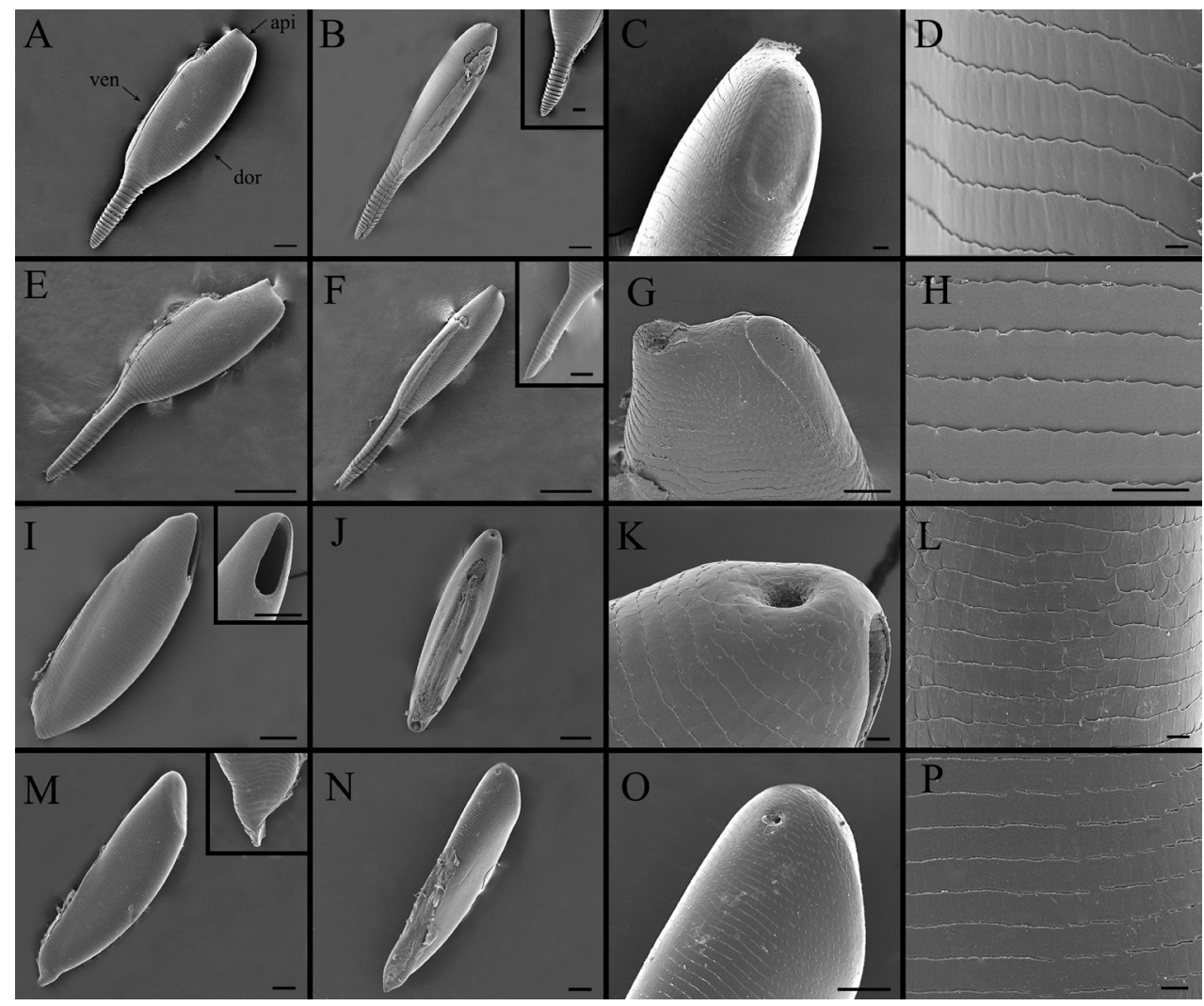

Figure I7. Right lateral $(\mathbf{A}, \mathbf{E}, \mathbf{I}, \mathbf{M})$ and ventral $(\mathbf{B}, \mathbf{F}, \mathbf{J}, \mathbf{N})$ view, micropyle $(\mathbf{C}, \mathbf{G}, \mathbf{K}, \mathbf{O})$ and ultrastructural details of plastron $(\mathbf{D}, \mathbf{H}, \mathbf{L}, \mathbf{P})$ of eggs in Gasterophilus species A-D G. flavipes (Olivier) E-H G. haemorrhoidalis (Linnaeus) I-L G. inermis (Brauer) M-P G. intestinalis. Abbreviations: api, apical; dor, dorsal; ven, ventral. Scale bars: $100 \mu \mathrm{m}(\mathbf{A}, \mathbf{B}, \mathbf{I}-\mathbf{J}, \mathbf{M}, \mathbf{- N}), 50 \mu \mathrm{m}$ (in box of B), $100 \mu \mathrm{m}$ (in box of I); $20 \mu \mathrm{m}(\mathbf{C}) ; 5 \mu \mathrm{m}(\mathbf{D}) ; 250 \mu \mathrm{m}(\mathbf{E}, \mathbf{F}), 20 \mu \mathrm{m}($ in box of F); $50 \mu \mathrm{m}(\mathbf{G}, \mathbf{O}) ; 25 \mu \mathrm{m}(\mathbf{H})$; $10 \mu \mathrm{m}(\mathbf{K}, \mathbf{L}, \mathbf{P})$.

Gastrus lativentris Brauer, 1858: 465. Type locality: Latvia, Curland (as "in Kurland gefangen").

Gastrus ferruginatus Zetterstedt, 1844: 978. Type locality: Sweden, Skåne, Tranås socken, Esperöd. (as "ad Esperöd in parœcia Tranås Scaniæ”).

Gasterophilus pecorum var. zebrae Rodhain \& Bequaert, 1920: 181. Type locality: Kenya and Tanzania.

Gastrophilus vulpecula Pleske, 1926: 227. Type locality: China, Inner Mongolia, Alxa League.

Gastrophilus gammeli Szilády, 1935: 140. Type locality: Hungary.

Gastrophilus hammeli: Paramonov 1940: 34, 46; incorrect subsequent spelling of gammeli Szilády, 1935.

Gastrophilus hummeli: Paramonov 194 "Dans les Pyrénées" 0: 32; incorrect subsequent spelling of gammeli Szilády, 1935.

Gastrus selysi Walker, 1849: 687. Nomen nudum. 


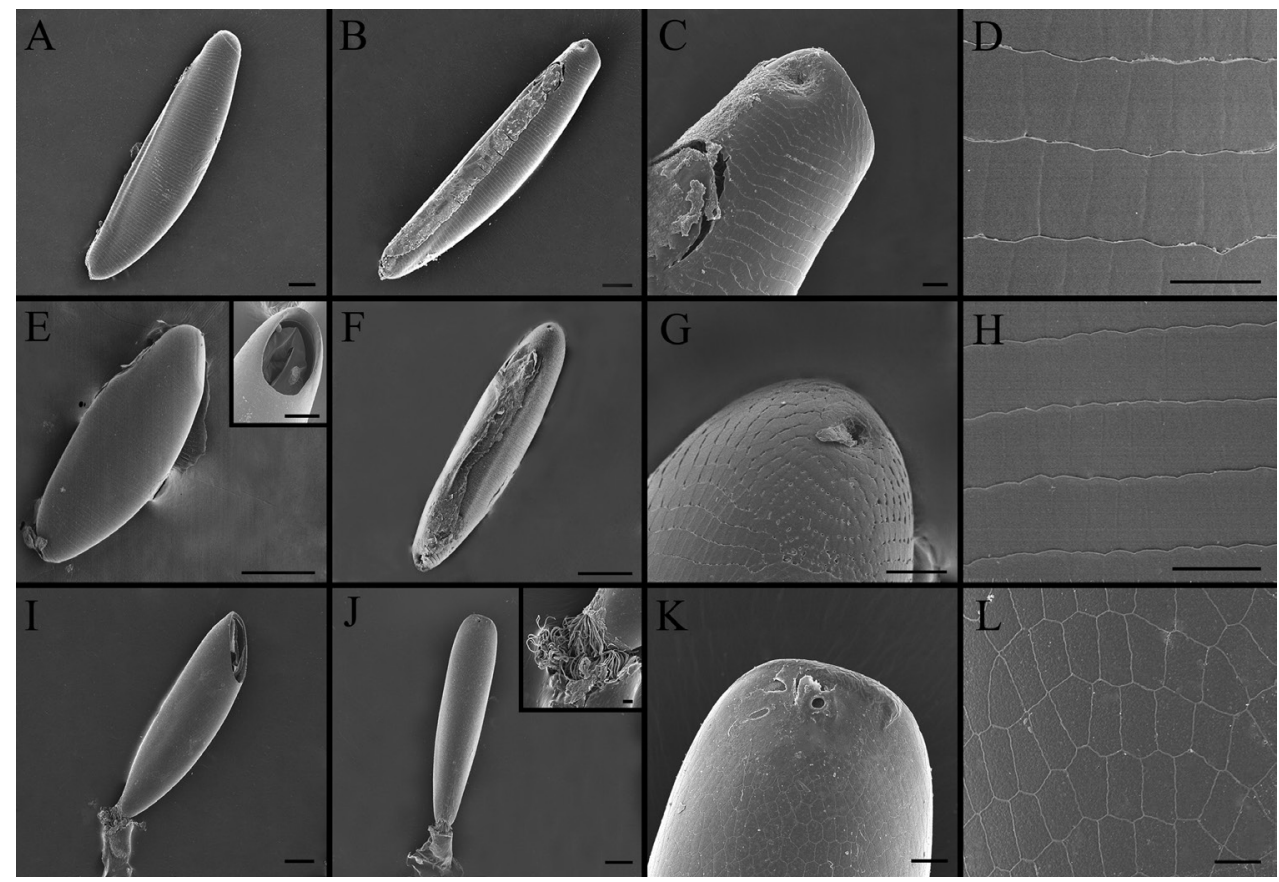

Figure 18. Right lateral $(\mathbf{A}, \mathbf{E}, \mathbf{I})$ and ventral $(\mathbf{B}, \mathbf{F}, \mathbf{J})$ view, micropyle $(\mathbf{C}, \mathbf{G}, \mathbf{K})$ and ultrastructural details of plastron (D, H, L) of eggs in Gasterophilus species A-D G. nasalis (Linnaeus) E-H G. nigricornis (Loew) I-L G. pecorum (Fabricius). Scale bars: $100 \mu \mathrm{m}$ (A, B, I-J), $10 \mu \mathrm{m}$ (in the box of J); $20 \mu \mathrm{m}(\mathbf{C}, \mathbf{H}$, $\mathbf{K}) ; 15 \mu \mathrm{m}(\mathbf{D}) ; 20 \mu \mathrm{m}(\mathbf{E}), 50 \mu \mathrm{m}$ (in the box of E); $150 \mu \mathrm{m}(\mathbf{F}) ; 25 \mu \mathrm{m}(\mathbf{G}) ; 10 \mu \mathrm{m}(\mathbf{L})$.

Selected references. Zumpt (1965: 114); Grunin (1969: 25); Pont (1973: 698); Soós and Minár (1986: 239); Cogley (1991b); Xue and Wang (1996: 2210); Otranto et al. (2005); Colwell et al. (2006: 5); Colwell et al. (2007); Zhang et al. (2016); Hoseini et al. (2017); Li et al. (2018); Yan et al. (2019).

Diagnosis. Antennal pedicel elongated, with length/width ratio more than 0.8 . Facial plate setose. Wing dark, with broad darkened patches with ill-defined edges; crossvein dm-cu absent. Meron with unmodified setae. Legs yellowish brown with femora distinctly darkened. Abdomen ground color yellow in male, mainly dark brown to black in female. Male cercus long and narrow, length/width ratio more than 3.0; surstylus yellow, with a rounded apex; processi longi elongated. Female sternite 8 with a longitudinal concavity in the middle and with a keel-shaped apex.

Material examined. CHINA - Xinjiang Uyghur Autonomous Region ・ 1 $\overbrace{}^{\Uparrow}$; Akesu; 25 Sep. 1958; collector unknown; IOZ •19; Bayingolin Mongol Autonomous Prefecture, Qiemo County; Aqiang; 3000 m; 20 Jul. 1988; X.Z. Zhang leg.; IOZ •

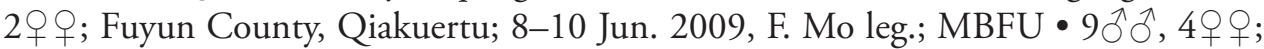
Kalamaili; 6 May-1 Jun. 2009; D. Zhang leg.; MBFU. - Inner Mongolia • 1q; Chifeng, Zhaowuda League, Right Banner; 22 Aug.-28 Sep. 1959; collector unknown;

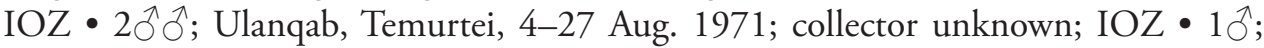
Xisuqi; 1 Sep. 1971; collector unknown; IOZ. 


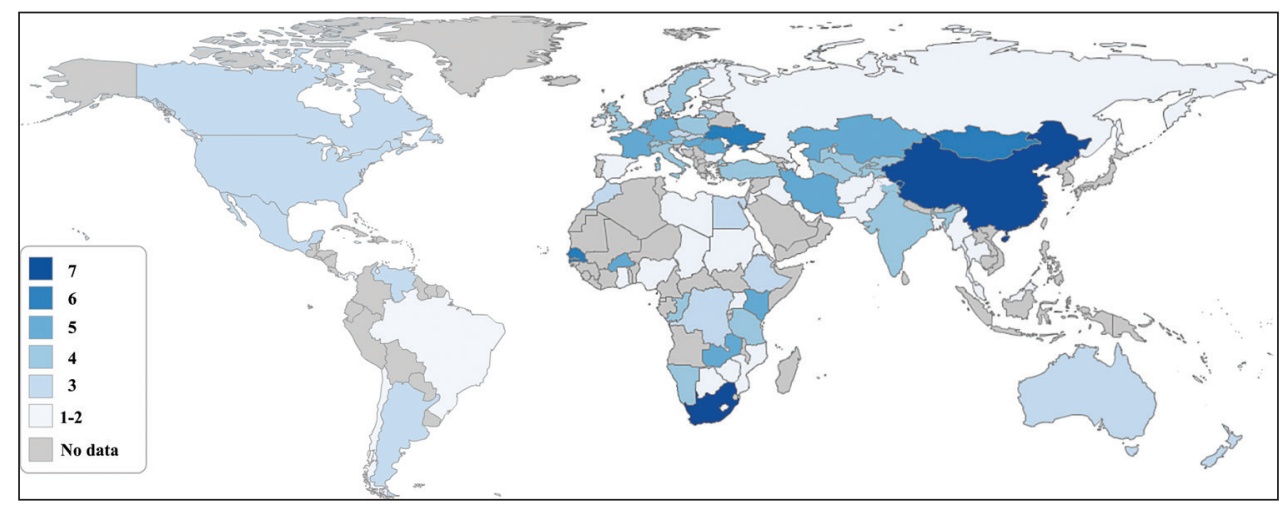

Figure 19. Species diversity map of all nine Gasterophilus species worldwide. Different colors represent the number of species recorded in a specific country. Interactive map showing the global distribution of all nine Gasterophilus species is available in Supplementary Information 1.

Hosts. Burchell's zebra (E. quagga burchellii), domestic horse (E. ferus caballus), donkey (E. africanus asinus), Mongolian wild ass (E. hemionus hemionus), Persian onager (E. hemionus onager Boddaert), wild horse (E. przewalskii).

Distribution. Afrotropical - Burkina Faso, Kenya, Namibia, Senegal, South Africa, Tanzania, Uganda, Zambia. Oriental - India. Palaearctic - Austria, Belgium, China (Heilongjiang, Inner Mongolia, Xinjiang), Czech Republic, Denmark, France, Germany, Hungary, Iran, Italy (incl. Corsica and Sicily), Latvia, Lithuania, Mongolia, Poland, Romania, Sweden, Switzerland, The Netherlands, Turkey, Ukraine, United Kingdom.

\section{Gasterophilus ternicinctus (Gedoelst, 1912)}

Figs 3G-I, 6E, F, 9G-I, 10I, 13G-I; Table 1

Gasterophilus ternicinctus Gedoelst, 1912: 426. Type locality: Democratic Republic of the Congo (as "Zaire"), $11.5 \mathrm{~km} \mathrm{~W}$ of Luapula river (as "6 milles W. du Luapula"). Gasterophilus gedoelsti Rodhain \& Bequaert, 1920: 188. Type locality: Kenya.

Selected references. Zumpt (1965: 128); Cogley (1991b); Colwell et al. (2006: 36).

Diagnosis. Facial plate bare. Wing with darkened patches with demarcated edges. Distance between crossveins $\mathrm{r}-\mathrm{m}$ and $\mathrm{dm}$-cu less than length of $\mathrm{r}-\mathrm{m}$. Meron with unmodified setae. Legs yellowish brown, with tibiae and tarsi more or less darkened. Hind trochanter of male with a long, spatulate process, of female with a tubercle; hind tibia and tarsus flattened distinctly in male, slightly in female; tarsomeres $2-4$ shortened in both sexes, broader than long. Abdomen ground color yellow in both male and female. Male cercus elongated and broad, length/width ratio around 1.5; surstylus mainly black with yellow coloration basally, and a rounded apex. Female abdominal segment 7 distinctly longer than broad, sternite 8 longitudinally ridged in the middle and with a scallop-shaped apex. 
Material examined. SOUTH AFRICA 1 1 ; KwaZulu; Hluhluwe-Imfolozi Park; 8 Mar. 1963; collector unknown; MBFU. KENYA 1 10, 1 \%; Kenplains, Athi river; 13 Mar. 1991; C.F. Dewhurst leg.; NHMUK.

Hosts. Burchell's zebra (E. quagga burchellii).

Distribution. Afrotropical - Burkina Faso, Democratic Republic of the Congo, Kenya, Republic of the Congo, Senegal, South Africa, Zambia.

\section{Acknowledgements}

We wish to thank Prof Jun Chen, Ms Hong Liu and Ms Kuiyan Zhang (Institute of Zoology, Chinese Academy of Sciences), Dr Olga Ovtshinnikova (Zoological Institute, Russian Academy of Sciences, St Petersburg) and Ms Tricia Pillay (KwaZulu-Natal Museum, Pietermaritzburg, South Africa) for kindly arranging for loans of Gasterophilus specimens, Mr Nigel Wyatt (Natural History Museum, London) and Mr Peter Sehnal (Naturhistorisches Museum Wien) for providing photos and locality details of particular specimens. This study was supported by the National Science Foundation of China (Nos. 31872964 and 31572305) and the Fundamental Research Funds for the Central Universities (No. 2019JQ0318) to D Zhang, Key Project of International Scientific and Technological Innovation Cooperation, National Key R\&D Program of China (No. 2016YFE0203100), and by an award from the State Scholarship Fund of China Scholarship Council (No. 201806510006) to X.-y. Li to pursue her studies at the University of Copenhagen.

\section{References}

Agassiz JLR (1846) Nomenclatoris zoologici index universalis: continens nomina systematica classium, ordinum, familiarum, et generum animalium omnium, tam viventium quam fossilium. Jent \& Gassmann, Switzerland, 393 pp.

Anderson JR (2006) Adult biology. In: Colwell DD, Hall MJR, Scholl PJ (Eds) The Oestrid Flies: Biology, Host-Parasite Relationships, Impact and Management. CABI, Wallingford, 140-166. https://doi.org/10.1079/9780851996844.0140

Bezdekova B, Jahn P, Vyskocil M (2007) Pathomorphological study on gastroduodenal ulceration in horses: Localisation of lesions. Acta Veterinaria Hungarica 55: 241-249. https:// doi.org/10.1556/AVet.55.2007.2.10

Bezzi M (1916) Un nuova specie di Estride dell'Eritrea. Bollettino del Laboratorio di zoologia generale e agraria della R. Scuola superiore d'agricoltura in Portici 10: 27-32.

Bigot JMF (1884) Descriptions de Diptères nouveaux récoltés par M. le professeur Magretti dans le Soudan oriental. Annales de la Société entomologique de France ser. 6, 4(2): 57-59.

Brauer F (1858) Neue Beiträge zur Kenntniss der europäischen Oestriden. Verhandlungen der Kaiserlich-Königliche Zoologisch-Botanischen Gesellschaft in Wien 8: 464. 
Brauer F (1863) Monographie der Oestriden. W. Braumüller, Wien, 292 pp. https://doi. org/10.5962/bhl.title.57896

Catts EP (1979) Hilltop aggregation and mating behavior by Gasterophilus intestinalis (Diptera: Gasterophilidae). Journal of Medical Entomology 16: 461-464. https://doi.org/10.1093/ jmedent/16.6.461

Clark B (1797) Observations on the genus Oestrus. Transactions of the Linnean Society of London 3: 289-329. https://doi.org/10.1111/j.1096-3642.1797.tb00570.x

Clark B (1815) An Essay on the Bots of Horses and Other Animals. Old Bailey, London, 72 pp. https://doi.org/10.5962/bhl.title. 159664

Clark B (1816) Discovery of the fly of the white bot. Supplementary sheet. London, 4 pp.

Cogley TP (1991a) Key to the eggs of the equid stomach bot flies Gasterophilus Leach 1817 (Diptera: Gasterophilidae) utilizing scanning electron microscopy. Systematic Entomology 16: 125-133. https://doi.org/10.1111/j.1365-3113.1991.tb00681.x

Cogley TP (1991b) Status of the stomach bot fly Gasterophilus lativentris (Brauer) 1858 (Diptera: Gasterophilidae). Systematic Entomology 16: 135-136. https://doi. org/10.1111/j.1365-3113.1991.tb00682.x

Cogley TP, Anderson JR, Cogley LJ (1982) Migration of Gasterophilus intestinalis larvae (Diptera: Gasterophilidae) in the equine oral cavity. International Journal for Parasitology 12: 473-480. https://doi.org/10.1016/0020-7519(82)90079-0

Cogley TP, Cogley MC (2000) Field observations of the host-parasite relationship associated with the common horse bot fly, Gasterophilus intestinalis. Veterinary Parasitology 88: 93105. https://doi.org/10.1016/S0304-4017(99)00191-0

Colwell DD, Hall MJR, Scholl PJ (2006) The Oestrid Flies: Biology, Host-Parasite Relationships, Impact and Management. CABI, Wallingford, 357 pp. https://doi. org/10.1079/9780851996844.0000

Colwell DD, Otranto D, Horak IG (2007) Comparative scanning electron microscopy of Gasterophilus third instars. Medical and Veterinary Entomology 21: 255-264. https://doi. org/10.1111/j.1365-2915.2007.00692.x

Coquillett DW (1910) The type-species of the North American genera of Diptera. Proceedings of the United States National Museum, Washington, 499-647. https://doi.org/10.5479/ si.00963801.37-1719.499

Colwell DD (2006) Life cycle strategies. In: Colwell DD, Hall MJR, Scholl PJ (Eds) The Oestrid Flies: Biology, Host-Parasite Relationships, Impact and Management. CABI, Wallingford, 67-77. https://doi.org/10.1079/9780851996844.0067

Cumming JM, Wood DM (2009) Adult morphology and terminology. In: Brown BV, Borkent A, Cumming JM (Eds), Manual of Central American Diptera Vol. 1. NRC Research Press, Ottawa, 9-50.

Curtis J (1826) British entomology: being illustrations and descriptions of the genera of insects found in Great Britain and Ireland: containing coloured figures from nature of the most rare and beautiful species, and in many instances of the plants upon which they are found. Printed for the author, London, 146 pp.

Dinulescu G (1932) Recherches sur la biologie des gastrophiles, anatomie, physiologie, cycle évolutif. Annales des Sciences Naturelles Zoologie Série 10: 183 pp. 
Dinulescu G (1938) Gastrophilus veterinus var. aureus. Archives Roumaines de Pathologie Expérimentale et de Microbiologie (Bucuresti) 11: 315-335.

Dove WE (1918) Some biological and control studies of Gastrophilus haemorrhoidalis and other bots of horses. United States Department of Agriculture 597: 52 pp. https://doi. org/10.5962/bhl.title.64448

Enderlein G (1934) Dipterologica. Sitzungsberichte der Gesellschaft naturforschender Freunde zu Berlin 1933: 416-429.

Escartin P, Bautista G (1993) Comparison of five tests for the serologic diagnosis of myiasis by Gasterophilus spp. larvae (Diptera: Gasterophilidae) in horses and donkeys: a preliminary study. Medical and Veterinary Entomology 7: 233-237. https://doi. org/10.1111/j.1365-2915.1993.tb00682.x

Fabricius JC (1787) Mantissa insectorum sistens eorum species nuper detectas adiectis characteribus genericis, differentiis specificis, emendationibus, observationibus. Impensis Christian Gottlob Proft, Copenhagen, 382 pp. https://doi.org/10.5962/bhl.title.36471

Fabricius JC (1794) Entomologia systematica emendata et aucta, Vol. 4: Secundum classes, ordines, genera, species. Impensis Christ Gottl Proft, Copenhagen, 472 pp.

Felix SR, Silva CE, Schmidtt E, Nizoli LQ, Götze MM, Silva SS (2007) Presence of Gasterophilus (Leach, 1817) (Diptera: Oestridae) in horses in Rio Grande do Sul State, Brazil. Parasitologia Latinoamericana 62: 122-126. https://doi.org/10.4067/S071777122007000200004

Ferrar PA (1987) Guide to the breeding habits and immature stages of Diptera Cyclorrhapha. In: Lyneborg L (Ed.) Entomonograph, Vol. 8. Scandinavian Science Press, Copenhagen, 907 pp.

Ganjali M, Keighobadi M (2016) A rare case of gastric Myiasis in a lion caused by Gasterophilus intestinalis (Diptera: Gasterophilidae)-case report. Journal of Arthropod-Borne Diseases 10: 423-425.

Gedoelst LM (1912) Contribution à la faune des Oestrides du Congo belge. Revue Zoologique Africaine 1: 426-432.

Gedoelst LM (1923) Note sur la larve du Gasterophilus haemorrhoidalis et description de la larve d'une nouvelle espèce africaine. Annales de Parasitologie 1: 269-275. https://doi. org/10.1051/parasite/1923013269

De Geer C (1776) Mémoires pour servir à l'histoire des insectes. Tome sixième. P. Hesselberg, Stockholm, 523 pp.

Getachew AM, Innocent G, Trawford AF, Reid SWJ, Love S (2012) Gasterophilosis: A major cause of rectal prolapse in working donkeys in Ethiopia. Tropical Animal Health and Production 44: 757-762. https://doi.org/10.1007/s11250-011-9961-7

Gistel J (1848) Naturgeschichte des Thierreichs für höheren Schulen. R. Hoffmann, Stuttgart, $216 \mathrm{pp}$.

Grunin KJ (1965) 64b. Hypodermatidae. In: Lindner E (Ed.) Die Fliegen der Paläarktischen Region 8. Schweizerbart'sche, Stuttgart, 153 pp.

Grunin KJ (1966) 64a'. Oestridae. In: Lindner E (Ed.) Die Fliegen der Paläarktischen Region 8. Schweizerbart'sche, Stuttgart, $96 \mathrm{pp}$.

Grunin KJ (1969) 64a. Gasterophilidae. In: Lindner E (Ed.) Die Fliegen der Paläarktischen Region 8. Schweizerbart'sche, Stuttgart, 66 pp. 
Guérin-Méneville FÉ (1827) Dictionnaire classique d'histoire naturelle. Paris, Rey et Gravier, LibraIries-Editeurs, Auai des Augustins, no. 55; Baudouin FrèreS, LibraIries-Editeurs, Imprimeurs de la société D’Histoire Naturelle, Rue de Vaugirard, no. 36, Paris, 634 pp.

Guimarães JH (1967) Family Gasterophilidae. In: A Catalogue of the Diptera of the Americas South of the United States. Departamento de Zoologia, Secretaria da Agricultura, São Paulo, 1-2.

Guimarães JH, Papavero N (1999) Myiasis in Man and Animals in the Neotropical Region; Bibliographic Database. Editora Plêiade, São Paulo, 306 pp.

Güiris ADM, Rojas HNM, Berovides A V., Sosa PJ, Pérez EME, Cruz AE, Chávez HC, Moguel AJA, Jimenez-Coello M, Ortega-Pacheco A (2010) Biodiversity and distribution of helminths and protozoa in naturally infected horses from the biosphere reserve "La Sierra Madre de Chiapas", México. Veterinary Parasitology 170: 268-277. https://doi. org/10.1016/j.vetpar.2010.02.016

Hadwen S, Cameron AE (1918) A contribution to the knowledge of the bot-flies, Gastrophilus intestinalis, DeG., G. haemorrhoidalis, L., and G. nasalis, L. Bulletin of Entomological Research 9: 91-106. https://doi.org/10.1017/S0007485300037858

Hall M, Wall R (1995) Myiasis of humans and domestic animals. Advances in parasitology 35: 257-334. https://doi.org/10.1016/S0065-308X(08)60073-1

Hoseini SM, Zaheri BA, Adibi MA, Ronaghi H, Moshrefi AH (2017) Histopathological study of esophageal infection with Gasterophilus pecorum (Diptera: Oestridae) in Persian Onager (Equus hemionus onager). Journal of Arthropod-Borne Diseases 11: 441-445.

Huang H, Chu H, Cao J, Bu L, Hu D, Zhang D, Li K (2017) Distribution of Gasterophilus (Diptera, Gasterophilidae) Myiasis Foci in Arid Desert Steppe: a case study of Kalamaili Mountain Ungulate Nature Reserve. Linye Kexue/Scientia Silvae Sinicae 53. https://doi.or g/10.11707/j.1001-7488.20171116

Huang H, Zhang B, Chu H, Zhang D, Li K (2016) Gasterophilus (Diptera, Gasterophilidae) infestation of equids in the Kalamaili Nature Reserve, China. Parasite 23: 1-36. https:// doi.org/10.1051/parasite/2016036

James MT (1974) The flies that cause myiasis in man. United States Government Printing Office, Washington, $175 \mathrm{pp}$.

Kaboret Y, Pangui LJ, Vercruysse J (1986) Note on gasterophilosis in donkeys in Burkina Faso. Revue d'Elevage et de Médecine Vétérinaire des Pays Tropicaux 39: 211-212.

Kettle P (1974) The genus Gasterophilus in the horse in New Zealand. New Zealand Veterinary Journal 22: 43-45. https://doi.org/10.1080/00480169.1974.34130

Leach WE (1817) On the arrangement of oestrideous insects. In: On the genera and species of eproboscideous insects, and on the arrangement of oestrideous insects. Neill \& Co., Edinburg, 1-20.

Leite ACR, Scott FB, Evangelista LG (1999) Scanning electron microscope observations on third-instar Gasterophilus nasalis (Diptera: Oestridae). Journal of Medical Entomology 36: 643-648. https://doi.org/10.1093/jmedent/36.6.643

Li XY, Chen Y, Wange Q, Li K, Pape T, Zhang D (2018) Molecular and morphological characterization of third instar Palaearctic horse stomach bot fly larvae (Oestridae: Gasterophilinae, Gasterophilus). Veterinary Parasitology 262: 56-74. https://doi.org/10.1016/j. vetpar.2018.09.011 
Li XY, Pape T, Zhang D (2019) Gasterophilus flavipes (Oestridae: Gasterophilinae): a horse stomach bot fly brought back from oblivion with morphological and molecular evidence. PLoS ONE 14(8): e0220820. https://doi.org/10.1371/journal.pone.0220820

Linnaeus C (1758) Systema naturae per regna tria naturae :secundum classes, ordines, genera, species, cum characteribus, differentiis, synonymis, locis. Salvii, Stockholm, 824 pp. https://doi.org/10.5962/bhl.title.542

Liu SH, Li K, Hu DF (2016) The incidence and species composition of Gasterophilus (Diptera, Gasterophilidae) causing equine myiasis in northern Xinjiang, China. Veterinary Parasitology 217: 36-38. https://doi.org/10.1016/j.vetpar.2015.12.028

Loew H (1863) Zwei neue europäische Dipterengattungen. Wiener Entomologische Monatschrift 7: 38-40.

Lucientes J (2002) Gasterophilidae. In: Carles-Tolrá M (Ed.) Catalogue of the Diptera of Spain, Portugal and Andorra (Insecta). Monografías SEA 8, 207 pp.

Macquart PJM (1843) Diptères exotiques nouveaux ou peu connus. Tome deuxième, $3^{\text {eme }}$ partie. Roret, Paris, 460 pp.

Mashayekhi M, Ashtari B (2013) Study of Gasterophilus role in Equine Gastric Ulcer Syndrome in Tabriz area. Bulletin of Environment, Pharmacology and Life Sciences 2: 169-172.

Meigen JW (1824) Systematische Beschreibung der bekannten europäischen zweiflügeligen Insekten. Vierter Theil. Schulz-Wundermann'sche Buchhandlung, Hamm, 428 pp. [pls. 33-41]

Muller B, Ranwashe F (2017) NMSA: Arthropod Collections (1900-2012). Version 1.1. South African National Biodiversity Institute. Occurrence dataset. https://doi.org/10.15468/a4tpcb van Noort DS, Ranwashe F (2017) IZIKO South Africa Museum Collection (1800-2013). Version 1.3. South African National Biodiversity Institute. Occurrence dataset. https:// doi.org/10.15468/vf3ko1

Olivier GA (1811) Encyclopédie méthodique. Histoire naturelle. Insectes. Vol. 8. H Agasse, Paris, $722 \mathrm{pp}$.

Otranto D, Milillo P, Capelli G, Colwell DD (2005a) Species composition of Gasterophilus spp. (Diptera, Oestridae) causing equine gastric myiasis in southern Italy: Parasite biodiversity and risks for extinction. Veterinary Parasitology 133: 111-118. https://doi.org/10.1016/j. vetpar.2005.05.015

Otranto D, Traversa D, Milillo P, De Luca F, Stevens J (2005b) Utility of Mitochondrial and Ribosomal Genes for Differentiation and Phylogenesis of Species of Gastrointestinal Bot Flies. Journal of Economic Entomology 98: 2235-2245. https://doi.org/10.1603/00220493-98.6.2235

Özdal N, Biçek K, Orunç Ö, Değer S (2010) Presence of Gasterophilus species in horses in Van Region. YYU Veteriner Fakultesi Dergisi 21: 87-90.

Pandey VS, Ouhelli H, Verhulst A (1992) Epidemiological observations on Gasterophilus intestinalis and $G$. nasalis in donkeys from Morocco. Veterinary Parasitology 41: 285-292. https://doi.org/10.1016/0304-4017(92)90087-P

Papavero N (1977) The World Oestridae (Diptera) Mammals and Continental Drift. Series Entomologica Vol. 14, 240 pp. https://doi.org/10.1007/978-94-010-1306-2 
Pape T (1996) Memoires of Entomology, Vol. 8. International Catalogue of the Sarcophagidae of the world. Stockholm, 558 pp.

Pape T (2001) Phylogeny of Oestridae (Insecta: Diptera). Systematic Entomology 26: 133171. https://doi.org/10.1046/j.1365-3113.2001.00143.x

Pape T (2013) Fauna Europaea: Gasterophilus. Fauna Europaea version 2019.10. https:// fauna-eu.org

Pape T, Piwczyński M, Wyborska D, Akbarzadeh K, Szpila K (2017) A new genus and species of hypodermatine bot flies (Diptera: Oestridae). Systematic Entomology 42: 387-398. https://doi.org/10.1111/syen.12220

Paramonov SJ (1940) Gastrophilidae und ihre Bekämpfungen. Kiew-Lwow, 128 pp.

Patton WS (1924) Gasterophilus crossi sp. nov., parasitic in its larval stage in the stomach of the horse in the Punjab. Indian Journal of Medical Research 11: 963.

Patton WS (1937) Studies on the higher Diptera of medical and veterinary importance. Annals of Tropical Medicine \& Parasitology 31: 351-359. https://doi.org/10.1080/00034983.19 37.11684990

Pearse B, Peucker S, Cottam R (1989) Identification of Gasterophilus haemorrhoidalis in Queensland. Australian Veterinary Journal 66: 380. https://doi.org/10.1111/j.1751-0813.1989. tb09745.x

Pillers AWN, Evans AM (1926) A new larva of Oestrus (Gastrophilus) from zebras. Annals of Tropical Medicine \& Parasitology 20: 263-266. https://doi.org/10.1080/00034983.1926 .11684499

Pilo C, Altea A, Scala A (2015) Gasterophilosis in horses in Sardinia (Italy): effect of meteorological variables on adult egg-laying activity and presence of larvae in the digestive tract, and update of species. Parasitology Research 114: 1693-1702. https://doi.org/10.1007/ s00436-015-4352-z

Pleske T (1926) Revue des espèces paléarctiques des Oestrides et catalogue raisonné de leur collection au Musée Zoologique de l'Académie des Sciences. Annuaire du Musée Zoologique de l'Académie Impériale des Sciences de Saint Pétersbourg 26: 215-230.

Pont AC (1973) Familiy Oestridae. In: Delfinado M, Hardy D (Eds) A Catalog of the Diptera of the Oriental Region: Suborder Cyclorrhapha. University Press of Hawaii, Honolulu, 700-701.

Pont AC (1980) Family Gasterophilidae. In: Crosskey RW (Ed.) Catalogue of the Diptera of the Afrotropical Region. British Museum of Natural History, London, 883-884.

Rodhain J, Bequaert J (1920) Oestrides d'antilopes et de zèbres recueillis en Afrique orientale avec un conspectus du genre Gasterophilus. Revue Zoologique Africaine 8: 169-228. https://doi.org/10.5962/bhl.part.22396

Rondani C (1857) Dipterologiae Italicae prodromus. Vol. 2. Species Italicae ordinis dipterorum in genera characteribus definita, ordinatim collectae, methodo analitica distinctae, et novis vel minus cognitis descriptis. Pars prima: Oestridae, Syrpfhidae, Conopidae. Stocchi, Parmae, 264 pp. https://doi.org/10.5962/bhl.title.8160

Schwab KC (1840) Die Oestraciden - Bremsen - der Pferde, Rinder und Schafe. Matthäus Pössenbacher, München, 83 pp. 
Sequeira JL, Tostes RA, Oliveira-Sequeira TCG (2001) Prevalence and macro- and microscopic lesions produced by Gasterophilus nasalis (Diptera: Oestridae) in the Botucatu Region, SP, Brazil. Veterinary Parasitology 102: 261-266. https://doi.org/10.1016/S03044017(01)00536-2

Soós Á, Minář J (1986) Family Gasterophilidae. In: Soós Á, Papp L (Eds) Catalogue of Palaearctic Diptera, Vol. 11. Scathophagidae - Hypodermatidae. Elsevier, Amsterdam, 237-240.

StatSilk (2018) StatPlanet Desktop: Interactive Data Visualization and Mapping Software http://www.statsilk.com

Sultanov MA (1951) A new species of botflies from horse -- Gastrophilus viridis sultanov sp. nov. Doklady Akademii Nauk Uzbekskoi SSR 1951: 41-44.

Szilády Z (1935) Die ungarischen Dasselfliegen. Állattani Közlemények 32: 136-140.

Tähtinen M, Lahti T (2017) Finnish Entomological Database. Version 1.2. Finnish Biodiversity Information Facility. Occurrence dataset. https://doi.org/10.15468/jlud8r

Tavassoli M, Bakht M (2012) Gastrophilus spp. myiasis in Iranian equine. Scientia Parasitologica 13: 83-86.

Townsend CHT (1918) New muscoid genera, species and synonymy (Diptera). Insecutor Inscitiae Menstruus 6: 157-182.

Townsend CHT (1934) New Neotropical oestromuscoid flies. Revista de Entomologia [Rio De Janeiro] 4: 201-212.

Walker F (1849) List of the specimens of dipterous insects in the collection of the British Museum. Part III. British Museum (Natural History), London, 485-687.

Wood DM (1987) Oestridae. In: McAlpine JF (Ed.) Manual of Nearctic Diptera, Vol. 2. Research Branch, Agriculture Canada, Ottawa, 1147-1158.

Wood DM (2006) Morphology of Adult Oestridae. In: Colwell DD, Hall MJR, Scholl PJ (Eds) The Oestrid Flies: Biology, Host-Parasite Relationships, Impact and Management. CABI, Wallingford, 79-80.

Xue WQ, Wang MF (1996) Gasterophilidae. In: Xue WQ, Zhao JM (Eds) Flies of China. Vol. 2. Liaoning Science and Technology Press, Shenyang, 2207-2215.

Yan L, Pape T, Elgar MA, Gao Y, Zhang D (2019) Evolutionary history of stomach bot flies in the light of mitogenomics. Systematic Entomology. https://doi.org/10.1111/syen.12356

Zetterstedt JW (1844) Diptera Scandinaviæ disposita et descripta. Tomus 3. Officina Lundbergiana, Lundae, 895-1280.

Zhang D, Li X, Liu X, Wang Q, Pape T (2016) The antenna of horse stomach bot flies: Morphology and phylogenetic implications (Oestridae, Gasterophilinae: Gasterophilus Leach). Scientific Reports 6. https://doi.org/10.1038/srep34409

Zhang D, Wang QK, Hu DF, Li K (2012) Sensilla on the antennal funiculus of the horse stomach bot fly, Gasterophilus nigricornis. Medical and Veterinary Entomology 26: 314-322. https://doi.org/10.1111/j.1365-2915.2011.01007.x

Zumpt F (1965) Myiasis of Man and Animals in the Old World. Butterworths Ltd., London, 264 pp.

Zumpt F, Paterson HE (1953) Studies on the family Gasterophilidae, with keys to the adults and maggots. Journal of the Entomological Society of Southern Africa 16: 59-72. 